\title{
Stability Boundary Analysis in Single-Phase Grid-Connected Inverters with PLL by LTP Theory
}

\author{
Valerio Salis, Student Member, IEEE, Alessandro Costabeber, Member, IEEE, Stephen M. Cox, \\ Pericle Zanchetta, Senior Member, IEEE, and Andrea Formentini, Member, IEEE
}

\begin{abstract}
Stability analysis of power converters in AC networks is complex due to the non-linear nature of the conversion systems. Whereas interactions of converters in DC networks can be studied by linearising about the operating point, the extension of the same approach to AC systems poses serious challenges, especially for single-phase or unbalanced three-phase systems. A general method for stability analysis of power converters suitable for single-phase or unbalanced AC networks is presented in this paper, based on Linear Time Periodic (LTP) theory. A singlephase grid-connected inverter with PLL is considered as case study. It is demonstrated that the stability boundaries can be precisely evaluated by the proposed method, despite the nonlinearity introduced by the PLL. Simulation and experimental results from a $10 \mathrm{~kW}$ laboratory prototype are provided to confirm the effectiveness of the proposed analysis.
\end{abstract}

Index Terms-Linear Time Periodic Systems, Harmonic State Space Model, Stability Analysis, Power Converters, PLL

\section{INTRODUCTION}

A C power systems based on power converters are complex and highly non-linear because both converters and loads often show a non-linear behaviour. Several practical cases of instability issues in such systems are discussed in the literature. For example, [1] reports an incident occurring on the Swiss railway power network due to the high level of harmonics generated by the interaction of new generation locomotive inverters. A more comprehensive review of potential instabilities affecting different railway systems is presented in [2]. In [3] it is stated that similar instability issues also affect LCC HVDC converters. Finally, [4] and [5] discuss several harmonic-related interactions, like the harmonics generated by the connection of wind and solar sources to the AC grid, causing resonances at low frequencies and leading to voltage and current distortions.

Based on these examples, it follows that a theory for stability analysis of such systems is required. Existing analysis techniques can be broadly categorized into frequency and time-domain methods. When a balanced and symmetric threephase AC system is considered, the analysis can be performed in the $d q$ reference frame [6], but this is no longer the

This paper is an extension of a conference paper (Stability analysis of single-phase grid-feeding inverters with PLL using Harmonic Linearisation and Linear Time Periodic (LTP) theory) presented at COMPEL, 27-30 June 2016, IEEE 17th Workshop on.

V. Salis, A. Costabeber, P. Zanchetta and A. Formentini are with the Department of Electrical and Electronic Engineering, University of Nottingham, Nottingham NG7 2RD, U.K. (e-mail: eexvs2@exmail.nottingham.ac.uk; alessandro.costabeber@nottingham.ac.uk; pericle.zanchetta@nottingham.ac.uk; andrea.formentini@nottingham.ac.uk).

S. M. Cox is with the School of Mathematical Sciences, University of Nottingham, Nottingham NG7 2RD, U.K. (e-mail: stephen.cox@nottingham.ac.uk)

Manuscript received ****, 2016; revised ****, 2016 case for unbalanced or single-phase systems. In such cases, one of the most popular frequency-domain method is the small-signal impedance-based stability criterion applied to the analysis of grid-connected power converters in the $a b c$ frame, [7]. The system is equivalently represented as a source and a load subsystem. Each system is described by its own small-signal impedances, which is calculated using Harmonic Linearisation techniques as shown in detail in [8], [9], [10]. Finally, stability of the system is assessed by applying the Nyquist criterion to the ratio of load to source small-signal impedances. However, potential limitations might affect this method, as discussed in [11], especially in the case where instabilities occur at frequencies below the fundamental grid frequency. An extension of this method is reported in [12], where a two-dimensional admittance for single-phase voltage source converters is calculated, able to capture the frequency interactions between load and source subsystem up to twice the line frequency, possibly overcoming the aforementioned limitations. However, if higher harmonic couplings are to be considered, the complex maths behind this method might be a limitation.

Another frequency-domain method is the dynamic phasor approach [13], where the 2-dimensional source and load impedances are evaluated and the system stability is assessed using the Generalised Nyquist Criterion [14]. This analysis relies on the assumption that all the signals in the system have a dominant first harmonic component, such that the DC and the higher components are neglected. This might be a limitation for the analysis of systems where the second or higher order harmonics play an important role.

The stability analysis method exploited in this paper is based on the theory presented in [15] and [16]. Such analysis can be performed both in the time domain, where the stability is assessed by evaluating the eigenvalues of the system, and in the frequency domain, where the Harmonic Transfer Function (HTF) operator is introduced and the LTP Nyquist Criterion is applied. Both time and frequency-domain approaches rely on the description of the system by its Harmonic State Space (HSS) model, which provides a linear relationship between the harmonic coefficients of input and output signals. Thus, the frequency cross-couplings are naturally described by the HSS model, which is a relevant feature compared to the previously discussed methods. The purpose of such a model is twofold. First it allows one to replace a non-linear circuit with its HSS model. Such a model requires less computation time in the simulation process and harmonic interaction and couplings are properly taken into account. For example, a HSS model of a controlled TCR is derived in [17], an HVDC converter and six-pulse STATCOM in [18], a single-phase 
grid-connected converter in [19] and a general method for modelling linear and switching subsystems in [20]. Second, based on a HSS model, stability analysis can be performed. In [21] and [22] stability analysis is performed on a single-phase grid-connected converter in the continuous time-domain, and in [23] the analysis is carried out in the discrete time-domain based on linearised models of the system, but non-linearities due to the PLL are not included in the analysis. In [24] the non-linear dynamics introduced by the PLL is included in the analysis using a small-signal model and an impedancebased stability analysis is performed but precise stability boundaries are not provided. In [25] a rigorous method based on eigenvalue analysis and LTP theory is presented, however the digital computation delay, $\mathrm{ZOH}$ and PWM dynamics are not included and experimental results are not provided.

In this paper, the time-domain approach based on the HSS model of a grid connected single-inverter with PLL and DC bus represented by an equivalent DC source is investigated in detail. To provide a comprehensive evaluation of the proposed method, the analysis is performed both in the continuous and in the discrete time-domain, leading in both cases to accurate results. It is worth mentioning that the primary objective of the analysis is to highlight the impact of non-linearities, i.e. the PLL, on system stability. Therefore, high frequency modelling is out of the scope of this work. Nevertheless, computation delay and sampling and hold have been included in the analysis for completeness. Starting from the non-linear average state space model, linearisation is applied in order to derive a linearised model, obtaining an LTP system. Then LTP theory is applied and stability analysis is performed based on the eigenvalue loci of the system. This paper demonstrates that, if properly applied, the method allows an accurate identification of the stability boundaries of the system, i.e. the stability threshold where the system moves from stable to unstable operation. To limit the complexity of the discussion, the stability threshold is analysed as the amplitude of the current injected in the AC grid, $I_{\text {ref }}$, increases. This simple choice has been made in order to highlight an instability effect that a simpler low-frequency linearisation approach for the PLL, used in conjunction with standard LTI stability analysis techniques, would not be able to identify. In fact, stability of a small-signal LTI model is independent of the amplitude of the control references. Even though previous literature exists on LTP theory applied to power converters, this work shows how the potential of the method can be exploited, guiding the reader through the theoretical procedure for stability analysis and quantifying the accuracy of the method. The paper is organized as follows: Section II provides a description of the system; Section III presents the continuous-time LTP stability analysis and Section IV the discrete-time one; in Section V analytical, simulation and experimental results for a $10 \mathrm{~kW}$ laboratory prototype are presented for different values of the grid inductor and of the damping resistor, showing in all cases good agreement with the expected stability boundaries; in Sections VI a comparison between continuous LTP and discrete LTP is provided.

\section{Single-Phase Grid-CONnected InVERTER With PLL MODEL}

The system under study is a current controlled gridconnected inverter with PLL, as shown in Fig. 1. The singlephase inverter is supplied by a DC source, $V_{d c}$, and its output is connected through an $L_{2} C_{1} L_{1}$ low-pass filter to the grid, which in turn is represented by an ideal sinusoidal voltage source, $V_{g}(t)=V_{g} \sin \left(\omega_{g} t\right)$, in series with an $R_{g} L_{g}$ impedance. Unity power factor operation is considered. A Phase Locked Loop (PLL) is used to measure the phase of the grid voltage and to generate the current reference signal, $I_{r e f}$, for the inverter output current, $I_{L 2}(t)$. A PI controller is then used to control the inverter.

TABLE I: Continuous-time system parameters

\begin{tabular}{|c|c|c|c|}
\hline$V_{g}=115 \sqrt{2} \mathrm{~V}$ & Volt. grid ampl. & $f_{g}=50 \mathrm{~Hz}$ & Volt. grid freq. \\
\hline$f_{p w m}=10 \mathrm{kHz}$ & Freq. carrier PWM & $V_{d c}=250 \mathrm{~V}$ & DC voltage \\
\hline$L_{1}=0 \mathrm{H}$ & Filter inductor & $R_{L 1}=0 \Omega$ & Filter res. \\
\hline$L_{2}=0.87 \mathrm{mH}$ & Filter inductor & $R_{L 2}=0.2 \Omega$ & Filter res. \\
\hline$L_{g}=2.2-2.95 \mathrm{mH}$ & Grid inductor & $R_{g}=0.4 \Omega$ & Grid res. \\
\hline$C_{1}=24 \mu \mathrm{F}$ & Filter capacitor & $R_{C 1}=0.6-1.4 \Omega$ & Damping res. \\
\hline$k_{p 1}=0.0581$ & \multirow{2}{*}{ Current PI param. } & $k_{p 2}=27.207$ & \multirow{2}{*}{ PLL PI param. } \\
\cline { 3 - 4 } & $k_{i 2}=493.48$ & \\
\hline$k_{i 1}=23.5$ & Ampl. current ref. & $T_{x}=50 \mu \mathrm{s}$ & Sampl. time \\
\hline$I_{r e f}=8-14 \mathrm{~A}$ & Sampl. freq. & $\gamma_{2}=-40000$ & Coeff. $\gamma_{2}(3)$ \\
\hline$f_{x}=2 f_{p w m}$ & Coeff. $\gamma_{1}(3)$ & $\gamma_{0}=0$ & Coeff. $\gamma_{0}(3)$ \\
\hline$\gamma_{1}=1.6 \mathrm{e}+09$ & Coeff. $\sigma_{2}(3)$ & $\sigma_{1}=1.6 \mathrm{e}+09$ & Coeff. $\sigma_{1}(3)$ \\
\hline$\sigma_{2}=80000$ & \multicolumn{2}{|}{}
\end{tabular}

It will be shown that the parameter $I_{\text {ref }}$, which is related to the amount of power that the inverter injects into the grid, has a threshold value, above which the system becomes unstable. That is, the instability is due to the fact that the PLL is no longer able to generate the correct phase reference for the controller. It is worth noticing that a damping resistor $R_{c}$ is used in the low-pass filter in order to simplify the design of the current control and focus on the analysis and quantification of the instability caused by the PLL.

For single-phase applications, the PLL needs to estimate the in-quadrature component of the grid voltage, $V_{o}(t)$, and among the several available options a linear filter that introduces a $T_{g} / 4$ delay at $\omega_{g}$ is used in this paper: $D(s)=$ $\omega_{g}^{2} /\left(s^{2}+s \omega_{g}+\omega_{g}^{2}\right)$. In Fig.1 the controllers are assumed to be implemented in the continuous-time domain, but the actual experimental converter has a digital controller. In the continuous-time analysis, in order to accurately reflect the characteristics of the digital control while maintaining the lower complexity of a continuous-time system for stability analysis, the computation delay $\left(T_{x}\right)$ with the zero-order hold ( $\mathrm{ZOH})$ delay of the pulse width modulator (PWM), (0.5 $T_{x}$ ) are included in the analysis with their continuous-time equivalents [26], [27]. This is based on the rationale that PLL instability is expected at relatively low frequencies, where the impact of controller discretization can be neglected, but the computation delay can play a relevant role. The validity of this assumption has been demonstrated by the accurate match between theoretical analysis and experimental results, as shown in the following sections. In the discrete-time analysis, the computation delay is naturally embedded in the discretetime model. The LTP analysis is performed on the overall system, hence including the non-linearities due to the PLL, and the instability threshold is precisely evaluated. The theoretical 


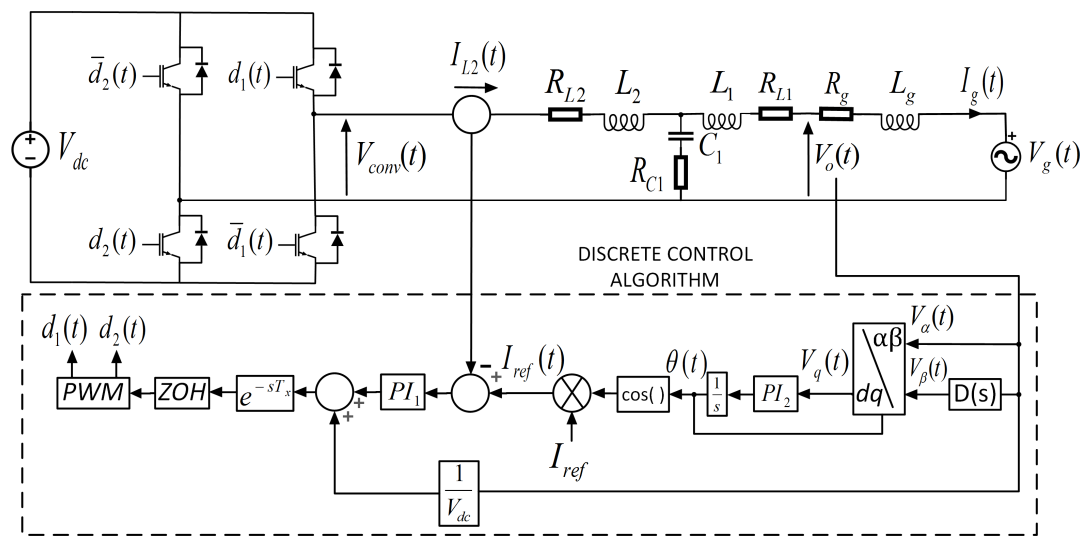

Fig. 1. Single-phase grid-connected inverter with PLL - switching model

analysis is based on the parameters that will later be used for the experimental prototype, summarised in Table I.

\section{Continuous Time-Domain Analysis}

\section{A. Continuous Non-Linear Time Periodic Average Model}

The proposed analysis is based on the average model of the system. Since the main focus of the analysis is the instability caused by the incorrect behaviour of the PLL and it is known that such instability arises at frequencies far below that of the switching [24], the switching model is replaced by the average one and the analysis is performed on the latter. The unit computation delay, the ZOH and the PWM blocks are represented in the continuous time domain by the following transfer function:

$$
H(s)=e^{-s T_{x}}\left[1-e^{-s T_{x}}\right] /\left(s T_{x}\right)
$$

The complex exponential is replaced with a first-order Padé approximation:

$$
e^{-s T_{x}}=\left(n_{1} s+n_{0}\right) /\left(d_{1} s+d_{0}\right)
$$

Substituting (2) in (1) gives the transfer function:

$$
H(s)=\left(\gamma_{2} s^{2}+\gamma_{1} s+\gamma_{0}\right) /\left(s^{3}+\sigma_{2} s^{2}+\sigma_{1} s\right)
$$

which relates the output of the current controller to the duty cycle. The whole system is equivalently represented by the state-space model (4), which is an $11^{\text {th }}$ order Continuous NonLinear Time Periodic (NLTP) system, with all the state-space variables $T_{g}$-periodic and the non-linearity due to the presence of the PLL. The state-space variables $x_{1}(t)-x_{4}(t)$ describe the internal dynamics of the PLL; $x_{5}(t)$ is the state-space variable associated to the current control PI; $x_{6}(t)$ represents the grid current, $I_{g}(t) ; x_{7}(t)$ the inductor current, $I_{L 2}(t)$; $x_{8}(t)$ the voltage across the capacitor; $x_{9}(t)-x_{11}(t)$ the internal dynamics of the computation delay, ZOH and PWM. The only input signal of the state-space model is the input voltage $V_{g}(t)$.

$$
\begin{aligned}
V_{o}(t)= & {\left[\left(L_{1} R_{g}-L_{g}\left(R_{C 1}+R_{L 1}\right)\right) x_{6}(t)+L_{g} R_{C 1} x_{7}(t)\right.} \\
& \left.+L_{g} x_{8}(t)+L_{1} V_{g}(t)\right] /\left(L_{g}+L_{1}\right) \\
V_{\text {conv }}(t)= & V_{d c} \gamma_{0} x_{9}(t)+V_{d c} \gamma_{1} x_{10}(t)+V_{d c} \gamma_{2} x_{11}(t) \\
\dot{x}_{1}(t)= & x_{2}(t)
\end{aligned}
$$

$$
\begin{aligned}
\dot{x}_{2}(t)= & -\omega_{g}^{2} x_{1}(t)-\omega_{g} x_{2}(t)+\omega_{g}^{2} V_{o}(t) \\
\dot{x}_{3}(t)= & x_{4}(t)-k_{p 2} \sin \left(x_{3}(t)\right) V_{o}(t)+k_{p 2} \cos \left(x_{3}(t)\right) x_{1}(t) \\
\dot{x}_{4}(t)= & -k_{i 2} \sin \left(x_{3}(t)\right) V_{o}(t)+k_{i 2} \cos \left(x_{3}(t)\right) x_{1}(t) \\
\dot{x}_{5}(t)= & I_{r e f} \cos \left(x_{3}(t)\right)-x_{7}(t) \\
\dot{x}_{6}(t)= & {\left[-\left(R_{C 1}+R_{L 1}+R_{g}\right) x_{6}(t)+R_{C 1} x_{7}(t)+x_{8}(t)\right.} \\
& \left.-V_{g}(t)\right] /\left(L_{g}+L_{1}\right) \\
\dot{x}_{7}(t)= & {\left[R_{C 1} x_{6}(t)-\left(R_{C 1}+R_{L 2}\right) x_{7}(t)-x_{8}(t)\right.} \\
& \left.+V_{\text {conv }}(t)\right] /\left(L_{2}\right) \\
\dot{x}_{8}(t)= & {\left[-x_{6}(t)+x_{7}(t)\right] /\left(C_{1}\right) } \\
\dot{x}_{9}(t)= & x_{10}(t), \dot{x}_{10}(t)=x_{11}(t) \\
\dot{x}_{11}(t)= & -\sigma_{1} x_{10}(t)-\sigma_{2} x_{11}(t)+k_{i 1} x_{5}(t) \\
& +k_{p 1} I_{\text {ref }} \cos \left(x_{3}(t)\right)-k_{p 1} x_{7}(t)+V_{o}(t) / V_{d c}
\end{aligned}
$$

\section{B. Review of Continuous Linear Time Periodic Systems Theory}

Average models of real AC systems are usually NLTP, as shown in (4). Therefore, the first step is to linearise the system about its steady-state operating trajectory, reducing it to an LTP system for small perturbations, in contrast to DC systems where linearisation is applied about a constant steady-state operating point. Given a general Continuous NLTP system which is $T$-periodic:

$\dot{x}(t)=f(x(t))+g(x(t)) u(t), y(t)=h(x(t))+l(x(t)) u(t)$

and given a steady-state input $\bar{u}(t)$, this system is solved numerically (in Matlab for example) and the steady-state solution, $\bar{x}(t)$, is obtained. Now linearisation is performed, so a small-signal perturbation is added to each input, output and state, i.e. $u(t)=\bar{u}(t)+\tilde{u}(t), y(t)=\bar{y}(t)+\tilde{y}(t)$ and $x(t)=\bar{x}(t)+\tilde{x}(t)$. These are substituted into (5) and taking into account only the perturbation terms gives the linearised model, which results in a Continuous LTP system:

$$
\dot{\tilde{x}}(t)=A(t) \tilde{x}(t)+B(t) \tilde{u}(t), \tilde{y}(t)=C(t) \tilde{x}(t)+D(t) \tilde{u}(t)
$$

with the matrices $A(t), B(t), C(t)$ and $D(t)$ being $T$-periodic. The test signal for LTP systems is the Exponentially Modulated Periodic (EMP) signal [15], [16]. When an EMP signal is injected as test signal into an LTP system, it can be shown that 
both input and output spaces are the same and they include the same set of harmonic components. Thus:

$$
\begin{aligned}
& q(t)=e^{j \Omega t} \sum_{n=-\infty}^{+\infty} q_{n} e^{j n \omega_{T} t} \text { for } q=u, x, y \\
& \dot{x}(t)=\sum_{n=-\infty}^{+\infty}\left(j \Omega+j n \omega_{T}\right) x_{n} e^{j\left(\Omega+n \omega_{T}\right) t}
\end{aligned}
$$

By substituting (7)-(8) in (6), expanding the matrices $A(t)$, $B(t), C(t)$ and $D(t)$ in Fourier series and using the Cauchy product theorem, it is possible to write:

$$
\begin{aligned}
\left(j \Omega+j n \omega_{T}\right) x_{n} & =\sum_{m=-\infty}^{\infty} A_{n-m} x_{m}+\sum_{m=-\infty}^{\infty} B_{n-m} u_{m} \\
y_{n} & =\sum_{m=-\infty}^{\infty} C_{n-m} x_{m}+\sum_{m=-\infty}^{\infty} D_{n-m} u_{m}
\end{aligned}
$$

This is a concise representation of the input-output relationship between the Fourier coefficients of the input and output signals. However, the manipulation of Fourier series usually leads to complicated calculation and for this reason the Toeplitz transform is introduced to simplify the analysis.

A Toeplitz transformation is defined as follows:

$$
\mathcal{T}[A(t)]=\mathcal{A}=\left[\begin{array}{ccccc}
\ddots & \vdots & \vdots & \vdots & \\
\cdots & A_{0} & A_{-1} & A_{-2} & \cdots \\
\cdots & A_{1} & A_{0} & A_{-1} & \cdots \\
\cdots & A_{2} & A_{1} & A_{0} & \cdots \\
& \vdots & \vdots & \vdots & \ddots
\end{array}\right]
$$

which is a doubly infinite block Toeplitz matrix and the matrices $A_{i}$ are the Fourier matrix coefficients of the $T$ periodic matrix $A(t)$. A similar definition applies to the matrices $\mathcal{T}[B(t)]=\mathcal{B}, \mathcal{T}[C(t)]=\mathcal{C}, \mathcal{T}[D(t)]=\mathcal{D}$ and to the vectors $\mathcal{T}[x(t)]=\mathcal{X}, \mathcal{T}[u(t)]=\mathcal{U}, \mathcal{T}[y(t)]=\mathcal{Y}$. Consider now the system of equations (9), which apply for all $n$. The Toeplitz transform is used in order to obtain a clearer and more compact notation. Thus the Harmonic State Space Model (HSSM) of the LTP system that follows from (6) is as follows:

$$
s \mathcal{X}=(\mathcal{A}-\mathcal{N}) \mathcal{X}+\mathcal{B U}, \mathcal{Y}=\mathcal{C} \mathcal{X}+\mathcal{D U}
$$

with $\mathcal{N}=\operatorname{diag}\left(\ldots, N_{-n}, \ldots, N_{-1}, N_{0}, N_{1}, \ldots, N_{n}, \ldots\right)$ and $N_{n}$ a diagonal matrix of the same dimensions as $A_{n}$ with diagonal coefficients equal to $j n \omega_{T}$. Through simple steps similar to the analysis of LTI systems, the Harmonic Transfer Function (HTF) of the system then follows from (11):

$$
\mathcal{Y}=\hat{G}(s) \mathcal{U}, \hat{G}(s)=\mathcal{C}[s \mathcal{I}-(\mathcal{A}-\mathcal{N})]^{-1} \mathcal{B}+\mathcal{D}
$$

Stability analysis can now be performed evaluating the eigenvalues of the matrix $(\mathcal{A}-\mathcal{N})$. If all the eigenvalues have $\operatorname{Re}\left[\lambda_{i}\right] \leq 0$, where those with $\operatorname{Re}\left[\lambda_{i}\right]=0$ have algebraic multiplicity equal to 1 , then the system is stable, otherwise the system is unstable.

So far an infinite number of coefficients has been considered in the Fourier series expansion. In the practical implementation of the analysis, a truncation order $N$ is introduced, which

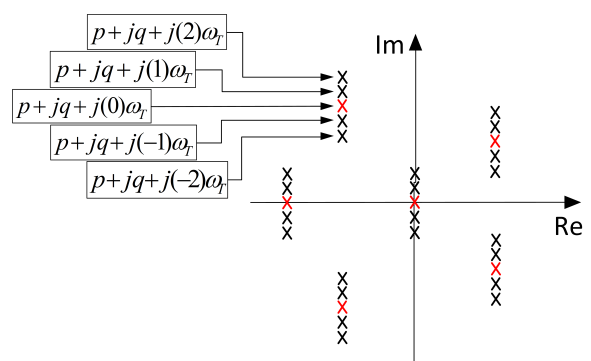

Fig. 2. General eigenvalue loci of an LTP system: red - important eigenvalues; black - translated copies

refers to the maximum harmonic number taken into account. If $N=2$, for example, this means that the DC-component and the first and second harmonics are considered. The corresponding Fourier expansion involves the Fourier coefficients for $n=-2,-1,0,1,2$ :

$$
A(t)=\sum_{n=-N}^{N} A_{n} e^{j n \omega_{T} t}=\sum_{n=-2}^{2} A_{n} e^{j n \omega_{T} t}
$$

and the following associated truncated Toeplitz form is considered:

$$
\mathcal{T}[A(t)]=\mathcal{A}=\left[\begin{array}{ccccc}
A_{0} & A_{-1} & A_{-2} & Z & Z \\
A_{1} & A_{0} & A_{-1} & A_{-2} & Z \\
A_{2} & A_{1} & A_{0} & A_{-1} & A_{-2} \\
Z & A_{2} & A_{1} & A_{0} & A_{-1} \\
Z & Z & A_{2} & A_{1} & A_{0}
\end{array}\right]
$$

with $Z$ being a zero matrix of the same dimension as the $A_{n}$. So when the truncation order is increased a larger number of Fourier coefficients of the Fourier expansion is taken into account and at the same time the dimension of the associated Toeplitz form increases. Thus, given an LTP system of order $p$, i.e. with $p$ state-space variables, and a truncation order $N$, the number of eigenvalues associated with the matrix $\mathcal{A}-\mathcal{N}$ will be $(2 N+1) \times p$. However, only $p$ of these eigenvalues are relevant for stability analysis; all the others are translated copies of the original ones, with translation equal to $j n \omega_{T}$, $n= \pm 1, \ldots, \pm N$. Fig. 2 shows an example of LTP pole loci with $q=6$ and $N=2$. In red are depicted the important poles and in black their translated copies. It can be observed that for a large truncation order the eigenvalue loci result in long vertical lines of eigenvalues.

\section{Continuous Steady-State Solution}

As anticipated in the previous section, in order to apply linearisation the steady-state solutions of the system (4) must first be evaluated. We will discuss some mathematical considerations and then solve numerically the system of equations, exploiting the Harmonic Balance approach. It is worth mentioning that the detailed derivation is provided for rigour and completeness. An approximated steady-state solution might be used in practice to reduce the analytical burden. Knowing that the converter is operating in an AC system, the set of steadystate solutions will be of the form:

$$
\bar{x}_{i}(t)=\left|\bar{x}_{i}\right| \cos \left(\omega_{g} t+\arg \left(\bar{x}_{i}\right)\right)=\left(\bar{x}_{i} e^{j \omega_{g} t}+\text { c.c. }\right) / 2
$$




$$
\begin{aligned}
\text { for } i & =1,2,5,6,7,8,9,10,11 ; \bar{x}_{3}(t)=\omega_{g} t+\bar{x}_{03} \\
\bar{x}_{4}(t) & =\text { to be defined } ; \bar{V}_{x}(t)=\left|\bar{V}_{x}\right| \cos \left(\omega_{g} t+\arg \left(\bar{V}_{x}\right)\right) \\
& =\left(\bar{V}_{x} e^{j \omega_{g} t}+\text { c.c. }\right) / 2, x=o, \text { conv }, g
\end{aligned}
$$

where c.c. stands for the complex conjugate of the term preceding it within the square brackets. Substituting these expressions in the non-linear state-space model gives us:

$$
\begin{aligned}
\bar{x}_{5}= & \left(I_{r e f} e^{\left.j \bar{x}_{03}-\bar{x}_{7}\right) /\left(j \omega_{g}\right)}\right. \\
\bar{x}_{6}= & \frac{R_{C 1} \bar{x}_{7}+\bar{x}_{8}+j V_{g}}{R_{C 1}+R_{L 1}+R_{g}+j \omega_{g}\left(L_{g}+L_{1}\right)} \\
\bar{x}_{7}= & \frac{R_{C 1} \bar{x}_{6}-\bar{x}_{8}+\bar{V}_{c o n v}}{R_{C 1}+R_{L 2}+j \omega_{g} L_{2}} \\
\bar{x}_{8}= & \left(-\bar{x}_{6}+\bar{x}_{7}\right) /\left(j \omega_{g} C_{1}\right) \\
\bar{x}_{9}= & \bar{x}_{10} /\left(j \omega_{g}\right) \\
\bar{x}_{10}= & \bar{x}_{11} /\left(j \omega_{g}\right) \\
\bar{x}_{11}= & {\left[-\sigma_{1} \bar{x}_{10}-\sigma_{2} \bar{x}_{11}+k_{i 1} \bar{x}_{5}+k_{p 1} I_{r e f} e^{j \bar{x}_{03}}\right.} \\
& \left.-k_{p 1} \bar{x}_{7}+\bar{V}_{o} / V_{d c}\right] /\left(j \omega_{g}\right) \\
\bar{V}_{o}= & {\left[\left(L_{1} R_{g}-L_{g}\left(R_{C 1}+R_{L 1}\right)\right) \bar{x}_{6}+\left(L_{g} R_{C 1}\right) \bar{x}_{7}\right.} \\
& \left.+L_{g} \bar{x}_{8}+L_{1} V_{g} / j\right] /\left(L_{g}+L_{1}\right) \\
\bar{V}_{\text {conv }}= & V_{d c} \gamma_{0} \bar{x}_{9}+V_{d c} \gamma_{1} \bar{x}_{10}+V_{d c} \gamma_{2} \bar{x}_{11}
\end{aligned}
$$

These nine equations can now be solved numerically and the steady state solutions $\bar{x}_{5}, \bar{x}_{6}, \bar{x}_{7}, \bar{x}_{8}, \bar{x}_{9}, \bar{x}_{10}, \bar{x}_{11}, \bar{V}_{o}, \bar{V}_{\text {conv }}$ obtained. Proceeding with the analysis gives:

$$
\bar{x}_{2}=j \omega_{g} \bar{x}_{1}, \bar{x}_{1}=-j \bar{V}_{o}
$$

So the solutions $\bar{x}_{1}, \bar{x}_{2}$ are given by:

$$
\begin{gathered}
\bar{x}_{1}(t)=\left|\bar{V}_{o}\right| \cos \left(\omega_{g} t+\angle \bar{V}_{o}-\pi / 2\right) \\
\bar{x}_{2}(t)=\omega_{g}\left|\bar{V}_{o}\right| \cos \left(\omega_{g} t+\angle \bar{V}_{o}\right)
\end{gathered}
$$

The last two quantities to be defined are $\bar{x}_{3}$ and $\bar{x}_{4}$ :

$$
\bar{x}_{3}(t)=\omega_{g} t+\bar{x}_{03}
$$

Hence:

$$
\begin{aligned}
\dot{\bar{x}}_{3}(t) & =\omega_{g}=\bar{x}_{4}(t) \\
& -k_{p 2} \sin \left(\omega_{g} t+\bar{x}_{03}\right)\left|\bar{V}_{o}\right| \cos \left(\omega_{g} t+\angle \bar{V}_{o}\right) \\
& +k_{p 2} \cos \left(\omega_{g} t+\bar{x}_{03}\right)\left|\bar{V}_{o}\right| \sin \left(\omega_{g} t+\angle \bar{V}_{o}\right)
\end{aligned}
$$

Applying trigonometric simplification gives us:

$$
\bar{x}_{4}(t)=\omega_{g}-k_{p 2} \sin \left(/ \bar{V}_{o}-\bar{x}_{03}\right)
$$

So it follows that $\dot{\bar{x}}_{4}(t)=0$. But from the state-space model, again using trigonometric simplifications, we have:

$$
\dot{\bar{x}}_{4}(t)=k_{i 2}\left[-\sin \left(\bar{x}_{3}(t)\right) \bar{V}_{o}(t)+\cos \left(\bar{x}_{3}(t)\right) \bar{x}_{1}(t)\right]
$$

And so:

$$
\dot{\bar{x}}_{4}(t)=-k_{i 2}\left|\bar{V}_{o}\right| \sin \left(\angle \bar{V}_{o}-\bar{x}_{03}\right)
$$

which implies: $\angle \bar{V}_{o}-\bar{x}_{03}=0$ or $\pm \pi$. In our case, $\angle \bar{V}_{o}=\bar{x}_{03}$, which gives the last two solutions:

$$
\bar{x}_{3}(t)=\omega_{g} t+\angle \bar{V}_{o}, \bar{x}_{4}(t)=\omega_{g}
$$

The steady-state periodic functions calculated in this subsections will now be used to derive the continuous LTP model of the system.

\section{Continuous Linearised Model}

Following the steps illustrated in the previous section, the Continuous LTP small-signal model (35) for perturbations to the steady-state operating point of the Continuous NLTP system (4) is derived, which is of the form $\dot{\tilde{x}}(t)=A(t) \tilde{x}(t)$, with $A(t)$ being a $T_{g}$-periodic matrix. In this particular case the only matrix coefficients of the Fourier series expansion which differ from zero are $A_{0}, A_{1}$ and $A_{-1}$; these are reported in (36), (37) with $A_{-1}=A_{1}^{*}$ (*=complex conjugate). This is due to the fact that the steady-state solutions for all the state variables are either DC or single-frequency $T_{g}$-periodic $\mathrm{AC}$. It is now possible to derive the Toeplitz form $\mathcal{A}$ and evaluate the eigenvalues of $\mathcal{A}-\mathcal{N}$ to determine whether the system is stable or not. The full derivation is not reported for the sake of brevity.

\section{Discrete Time-Domain Analysis}

\section{A. Discrete Non-Linear Time Periodic Average Model}

The stability analysis proposed in the previous Section is based on a simplified continuous-time model of the system. Another possibility is to perform the analysis based on a discrete-time model of the system, thus providing a direct representation of the digital implementation of the controllers in the DSP of the experimental set-up. However, from a stability point of view, both continuous and discrete-time approaches lead to an accurate identification of the stability boundaries, as it will be shown in Section V. First, the control algorithm implemented in the DSP is reported, which involves the discretization of the four continuous time blocks, i.e. current controller, linear filter for quadrature signal generation, PLL controller and integrator block. The $Z O H$ transformation is applied to the PI controller and to the integrator of the PLL:

$$
P L L(z)=Z O H\left[\frac{1}{s}\left(k_{p 2}+\frac{k_{i 2}}{s}\right)\right]=\frac{F_{1} z+F_{0}}{z^{2}+E_{1} z+E_{0}}
$$

Since the operating bandwidth of such a controller does not exceed the grid frequency $\omega_{g}=50 \mathrm{~Hz}$, it is not necessary to use a more precise transformation such as that of Tustin and the direct relationship between input and output is avoided, which would have caused an algebraic loop. The remaining control blocks are discretized with the Tustin transformation, which gives:

$$
\begin{gathered}
P I_{1}(z)=\text { Tustin }\left[k_{p 1}+\frac{k_{i 1}}{s}\right]=D_{1}+\frac{D_{0}}{z+C_{0}} \\
D(z)=\text { Tustin }\left[\frac{\omega_{g}^{2}}{s^{2}+\omega_{g} s+\omega_{g}^{2}}\right]=B_{2}+\frac{B_{1} z+B_{0}}{z^{2}+A_{1} z+A_{0}}
\end{gathered}
$$

Next, the output $L C L$ filter and the equivalent grid are first described by the continuous-time state-space model (43), which is then discretized applying the $Z O H$ transformation, with the discrete state-space representation of the $L C L$ filter 


$$
\begin{aligned}
\dot{\tilde{x}}_{1}(t) & =\tilde{x}_{2}(t), \dot{\tilde{x}}_{2}(t)=-\omega_{g}^{2} \tilde{x}_{1}(t)-\omega_{g} \tilde{x}_{2}(t)+\omega_{g}^{2}\left(L_{1} R_{g}-L_{g}\left(R_{C 1}+R_{L 1}\right)\right) /\left(L_{g}+L_{1}\right) \tilde{x}_{6}(t) \\
& +\omega_{g}^{2} L_{g} R_{C 1} /\left(L_{g}+L_{1}\right) \tilde{x}_{7}(t)+\omega_{g}^{2} L_{g} /\left(L_{g}+L_{1}\right) \tilde{x}_{8}(t) \\
\dot{\tilde{x}}_{3}(t) & =\tilde{x}_{4}(t)-k_{p 2} \sin \left(\bar{x}_{3}(t)\right)\left(L_{1} R_{g}-L_{g}\left(R_{C 1}+R_{L 1}\right)\right) /\left(L_{g}+L_{1}\right) \tilde{x}_{6}(t)-k_{p 2} \sin \left(\bar{x}_{3}(t)\right) L_{g} R_{C 1} /\left(L_{g}+L_{1}\right) \tilde{x}_{7}(t) \\
& -k_{p 2} \sin \left(\bar{x}_{3}(t)\right) L_{g} /\left(L_{g}+L_{1}\right) \tilde{x}_{8}(t)-k_{p 2} \cos \left(\bar{x}_{3}(t)\right) \bar{V}_{o}(t) \tilde{x}_{3}(t)+k_{p 2} \cos \left(\bar{x}_{3}(t)\right) \tilde{x}_{1}(t)-k_{p 2} \sin \left(\bar{x}_{3}(t)\right) \bar{x}_{1}(t) \tilde{x}_{3}(t) \\
\dot{\tilde{x}}_{4}(t) & =-k_{i 2} \sin \left(\bar{x}_{3}(t)\right)\left(L_{1} R_{g}-L_{g}\left(R_{C 1}+R_{L 1}\right)\right) /\left(L_{g}+L_{1}\right) \tilde{x}_{6}(t)-k_{i 2} \sin \left(\bar{x}_{3}(t)\right) L_{g} R_{C 1} /\left(L_{g}+L_{1}\right) \tilde{x}_{7}(t) \\
& -k_{i 2} \sin \left(\bar{x}_{3}(t)\right) L_{g} /\left(L_{g}+L_{1}\right) \tilde{x}_{8}(t)-k_{i 2} \cos \left(\bar{x}_{3}(t)\right) \bar{V}_{o}(t) \tilde{x}_{3}(t)+k_{i 2} \cos \left(\bar{x}_{3}(t)\right) \tilde{x}_{1}(t)-k_{i 2} \sin \left(\bar{x}_{3}(t)\right) \bar{x}_{1}(t) \tilde{x}_{3}(t) \\
\dot{\tilde{x}}_{5}(t) & =-I_{r e f} \sin \left(\bar{x}_{3}(t)\right) \tilde{x}_{3}(t)-\tilde{x}_{7}(t) \\
\dot{\tilde{x}}_{6}(t) & =-\left(R_{C 1}+R_{L 1}+R_{g}\right) /\left(L_{g}+L_{1}\right) \tilde{x}_{6}(t)+R_{C 1} /\left(L_{g}+L_{1}\right) \tilde{x}_{7}(t)+1 /\left(L_{g}+L_{1}\right) \tilde{x}_{8}(t) \\
\dot{\tilde{x}}_{7}(t) & =R_{C 1} / L_{2} \tilde{x}_{6}(t)-\left(R_{C 1}+R_{L 2}\right) / L_{2} \tilde{x}_{7}(t)-1 / L_{2} \tilde{x}_{8}(t)+V_{d c} \gamma_{0} / L_{2} \tilde{x}_{9}(t)+V_{d c} \gamma_{1} / L_{2} \tilde{x}_{10}(t)+V_{d c} \gamma_{2} / L_{2} \tilde{x}_{11}(t) \\
\dot{\tilde{x}}_{8}(t) & =-1 / C_{1} \tilde{x}_{6}(t)+1 / C_{1} \tilde{x}_{7}(t), \dot{\tilde{x}}_{9}(t)=\tilde{x}_{10}(t), \dot{\tilde{x}}_{10}(t)=\tilde{x}_{11}(t) \\
\dot{\tilde{x}}_{11}(t) & =-\sigma_{1} \tilde{x}_{10}(t)-\sigma_{2} \tilde{x}_{11}(t)+k_{i 1} \tilde{x}_{5}(t)-k_{p 1} I_{r e f} \sin \left(\bar{x}_{3}(t)\right) \tilde{x}_{3}(t)-k_{p 1} \tilde{x}_{7}(t) \\
& +\left(L_{1} R_{g}-L_{g}\left(R_{C 1}+R_{L 1}\right)\right) /\left(V_{d c}\left(L_{g}+L_{1}\right)\right) \tilde{x}_{6}(t)+L_{g} R_{C 1} /\left(V_{d c}\left(L_{g}+L_{1}\right)\right) \tilde{x}_{7}(t)+L_{g} /\left(V_{d c}\left(L_{g}+L_{1}\right)\right) \tilde{x}_{8}(t)
\end{aligned}
$$

$A_{0}=\left[\begin{array}{cccccc}0 & 1 & 0 & 0 & 0 & 0 \\ -\omega_{g}^{2} & -\omega_{g} & 0 & 0 & 0 & \omega_{g}^{2}\left[\frac{L_{1} R_{g}-L_{g}\left(R_{C 1}+R_{L 1}\right)}{L_{g}+L_{1}}\right] \\ 0 & 0 & -k_{p 2}\left|\bar{V}_{o}\right| & 1 & 0 & 0 \\ 0 & 0 & -k_{i 2}\left|\bar{V}_{o}\right| & 0 & 0 & 0 \\ 0 & 0 & 0 & 0 & 0 & 0 \\ 0 & 0 & 0 & 0 & 0 & -\frac{R_{C 1}+R_{g}+R_{L 1}}{L_{g}+L_{1}} \\ 0 & 0 & 0 & 0 & 0 & \frac{R_{C 1}}{L_{2}} \\ 0 & 0 & 0 & 0 & 0 & -\frac{1}{C_{1}} \\ 0 & 0 & 0 & 0 & 0 & 0 \\ 0 & 0 & 0 & 0 & 0 & 0 \\ 0 & 0 & 0 & 0 & k_{i 1} & \frac{L_{1} R_{g}-L_{g}\left(R_{C 1}+R_{L 1}\right)}{V_{d c}\left(L_{g}+L_{1}\right)}\end{array}\right.$

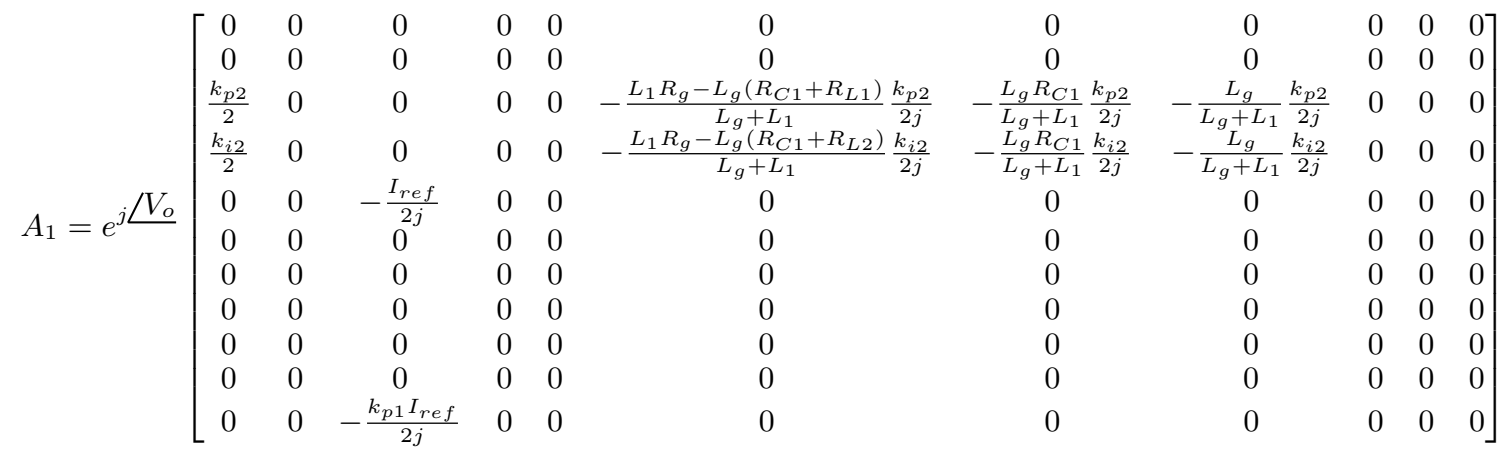

$$
\left[\begin{array}{c}
\dot{I}_{g}(t) \\
\dot{I}_{L 2}(t) \\
\dot{V}_{C 1}(t)
\end{array}\right]=\left[\begin{array}{ccc}
-\left(R_{C 1}+R_{g}\right) /\left(L_{g}+L_{1}\right) & R_{C 1} /\left(L_{g}+L_{1}\right) & 1 /\left(L_{g}+L_{1}\right) \\
R_{C 1} / L_{2} & -\left(R_{C 1}+R_{L 2}\right) / L_{2} & -1 / L_{2} \\
-1 / C_{1} & 1 / C_{1} & 0
\end{array}\right]\left[\begin{array}{c}
I_{g}(t) \\
I_{L 2}(t) \\
V_{C 1}(t)
\end{array}\right]+\left[\begin{array}{cc}
-1 /\left(L_{g}+L_{1}\right) & 0 \\
0 & 1 / L_{2} \\
0 & 0
\end{array}\right]\left[\begin{array}{c}
V_{g}(t) \\
V_{\text {conv }}(t)
\end{array}\right]
$$

an grid provided by the matrices $A^{L C L}$ and $B^{L C L}$, whose elements are the coefficients $a_{i j}^{L C L}$ and $b_{i j}^{L C L}$. Rearranging and making appropriate substitutions gives the Discrete NonLinear Time Periodic (DNLTP) average model of the system:

$$
\begin{aligned}
V_{\beta}(k) & =B_{0} x_{1}(k)+B_{1} x_{2}(k)+B_{2} V_{o}(k) \\
\theta(k) & =F_{0} x_{4}(k)+F_{1} x_{5}(k) \\
V_{o}(k) & =-R_{c} x_{6}(k)+R_{c} x_{7}(k)+x_{8}(k) \\
x_{1}(k+1) & =x_{2}(k) \\
x_{2}(k+1) & =-A_{0} x_{1}(k)-A_{1} x_{2}(k)+V_{o}(k)
\end{aligned}
$$

$$
\begin{aligned}
x_{3}(k+1) & =-C_{0} x_{3}(k)+I_{\text {ref }} \cos (\theta(k))-x_{7}(k) \\
x_{4}(k+1) & =x_{5}(k), x_{5}(k+1)=-E_{0} x_{4}(k) \\
& -E_{1} x_{5}(k)-\sin (\theta(k)) V_{o}(k)+\cos (\theta(k)) V_{\beta}(k) \\
x_{6}(k+1) & =a_{11}^{L C L} x_{6}(k)+a_{12}^{L C L} x_{7}(k)+a_{13}^{L C L} x_{8}(k) \\
& +b_{11}^{L C L} V_{g}(k)+b_{12}^{L C L} x_{9}(k) \\
x_{7}(k+1) & =a_{21}^{L C L} x_{6}(k)+a_{22}^{L C L} x_{7}(k)+a_{23}^{L C L} x_{8}(k) \\
& +b_{21}^{L C L} V_{g}(k)+b_{22}^{L C L} x_{9}(k) \\
x_{8}(k+1) & =a_{31}^{L C L} x_{6}(k)+a_{32}^{L C L} x_{7}(k)+a_{33}^{L C L} x_{8}(k)
\end{aligned}
$$




$$
\begin{aligned}
& +b_{31}^{L C L} V_{g}(k)+b_{32}^{L C L} x_{9}(k) \\
x_{9}(k+1) & =1 / V_{d c} V_{o}(k)+D_{0} x_{3}(k)+D_{1} I_{r e f} \cos (\theta(k)) \\
& -D_{1} x_{7}(k)
\end{aligned}
$$

In this model, the discrete state-space variables have the following meaning: $x_{1}, . ., x_{5}$ are associated to the discretized controllers, $x_{6}$ represents the grid current $I_{g}, x_{7}$ the inductor current $I_{L 2}, x_{8}$ the capacitor voltage $V_{C 1}$ and $x_{9}$ represents the control output, i.e. the duty cycle. The computational delay is naturally represented by the discrete state-space model, as it can be seen from equations $x_{6}(k+1), . ., x_{8}(k+1)$, where the control input $x_{9}(k)$ has been calculated based on the signals sampled at $(k-1) T_{x}$.

\section{B. Review of Discrete Linear Time Periodic Systems Theory}

Consider a general Discrete Non-Linear Time Periodic system:

$$
\begin{aligned}
x(k+1) & =f(x(k))+g(x(k)) u(k) \\
y(k) & =h(x(k))+l(x(k)) u(k),
\end{aligned}
$$

with period equal to $P$, i.e. $x(k)=x(k+P), u(k)=u(k+P)$ and $y(k)=y(k+P)$, and sampling time $T_{x}$. Choosing $T_{x}$ being a sub-multiple of the fundamental system period $T_{g}$, it follows that $P=T_{g} / T_{x}$. Linearising (45) around the $P$ periodic steady-state solution and considering only the first order terms gives the Discrete Linear Time Periodic (DLTP) system:

$$
\begin{aligned}
x(k+1) & =A(k) x(k)+B(k) u(k) \\
y(k) & =C(k) x(k)+D(k) u(k),
\end{aligned}
$$

with matrices $A(k), B(k), C(k)$ and $D(k)$ being $P$-periodic. As reported in [28], [29], the system described in (46) can be equivalently represented by a time-invariant model of the form:

$$
\begin{aligned}
q(k+1) & =\bar{A} q(k)+\bar{B} u(k) \\
y(k) & =\bar{C} q(k)+\bar{D} u(k),
\end{aligned}
$$

with $q(k)$ being a sampled version of $x(k)$, such that $q(k)=$ $x(k P)$, and the matrices $\bar{A}, \bar{B}, \bar{C}$ and $\bar{D}$ being constant. The most relevant feature for our purposes is the fact that stability can be equivalently assessed based on either (46) or (47). Since $\bar{A}$ can be easily calculated by:

$$
\bar{A}=A(k+P-1) \cdot A(k+P-2) \cdots A(k+1) \cdot A(k)
$$

stability analysis is performed based on the eigenvalue loci of $\bar{A}$. If all the eigenvalues of (48) lie inside the unit-circle, the system is stable, otherwise it is unstable. This shows how the discrete LTP analysis leads to a simpler procedure for stability assessment, avoiding Toeplitz transforms and without truncations.

\section{Discrete Steady-State Solution}

Now, with a similar approach to the one adopted in section III-(c), the steady-state solutions of the DNLTP model (44) are calculated, and only their general form is reported here for brevity:

$$
\begin{aligned}
\bar{x}_{i}(k) & =\left|\bar{x}_{i}\right| \cos \left(\omega_{g} k T_{x}+\arg \left(\bar{x}_{i}\right)\right)=\left(\bar{x}_{i} e^{j \omega_{g} k T_{x}}+c . c .\right) / 2 \\
\text { for } i & =1,2,3,6,7,8,9 ; \\
\bar{x}_{j}(k) & =x_{j 0}+x_{j 1} k T_{x} \text { for } i=4,5 .
\end{aligned}
$$

\section{Discrete Linearised Model}

Linearisation is performed around the calculated steadystate solutions and the discrete LTP small-signal model is given by the following set of equations:

$$
\begin{aligned}
\tilde{V}_{\beta}(k) & =B_{0} \tilde{x}_{1}(k)+B_{1} \tilde{x}_{2}(k)+B_{2} \tilde{V}_{o}(k) \\
\tilde{\theta}(k) & =F_{0} \tilde{x}_{4}(k)+F_{1} \tilde{x}_{5}(k) \\
\tilde{V}_{o}(k) & =-R_{c} \tilde{x}_{6}(k)+R_{c} \tilde{x}_{7}(k)+\tilde{x}_{8}(k) \\
\tilde{x}_{1}(k+1) & =\tilde{x}_{2}(k) \\
\tilde{x}_{2}(k+1) & =-A_{0} \tilde{x}_{1}(k)-A_{1} \tilde{x}_{2}(k)+\tilde{V}_{o}(k) \\
\tilde{x}_{3}(k+1) & =-C_{0} \tilde{x}_{3}(k)-I_{r e f} F_{0} \sin (\bar{\theta}(k)) \tilde{x}_{4}(k) \\
& -I_{r e f} F_{1} \sin (\bar{\theta}(k)) \tilde{x}_{5}(k)-\tilde{x}_{7}(k) \\
\tilde{x}_{4}(k+1) & =\tilde{x}_{5}(k) \\
\tilde{x}_{5}(k+1) & =-E_{0} \tilde{x}_{4}(k)-E_{1} \tilde{x}_{5}(k)-F_{0} \cos (\bar{\theta}(k)) \bar{V}_{o}(k) \tilde{x}_{4}(k) \\
& -F_{1} \cos (\bar{\theta}(k)) \bar{V}_{o}(k) \tilde{x}_{5}(k)-\sin (\bar{\theta}(k)) \tilde{V}_{o}(k) \\
& -F_{0} \sin (\bar{\theta}(k)) \bar{V}_{\beta}(k) \tilde{x}_{4}(k) \\
& -F_{1} \sin (\bar{\theta}(k)) \bar{V}_{\beta}(k) \tilde{x}_{5}(k)+\cos (\bar{\theta}(k)) \tilde{V}_{\beta}(k) \\
\tilde{x}_{6}(k+1) & =a_{11}^{L C L} \tilde{x}_{6}(k)+a_{12}^{L C L} \tilde{x}_{7}(k)+a_{13}^{L C L} \tilde{x}_{8}(k) \\
& +b_{12}^{L C L} \tilde{x}_{9}(k) \\
\tilde{x}_{7}(k+1) & =a_{21}^{L C L} \tilde{x}_{6}(k)+a_{22}^{L C L} \tilde{x}_{7}(k)+a_{23}^{L C L} \tilde{x}_{8}(k) \\
& +b_{22}^{L C L} \tilde{x}_{9}(k) \\
\tilde{x}_{8}(k+1) & =a_{31}^{L C L} \tilde{x}_{6}(k)+a_{32}^{L C L} \tilde{x}_{7}(k)+a_{33}^{L C L} \tilde{x}_{8}(k) \\
& +b_{32}^{L C L} \tilde{x}_{9}(k) \\
\tilde{x}_{9}(k+1) & =1 / V_{d c} \tilde{V}_{o}(k)+D_{0} \tilde{x}_{3}(k) \\
& -D_{1} I_{r e f} F_{0} \sin (\bar{\theta}(k)) \tilde{x}_{4}(k) \\
& -D_{1} I_{r e f} F_{1} \sin (\bar{\theta}(k)) \tilde{x}_{5}(k)-D_{1} \tilde{x}_{7}(k)
\end{aligned}
$$

This model is of the form $\tilde{x}(x+1)=A(k) \tilde{x}(k)$, and stability can be assessed by evaluating the discrete LTP eigenvalue loci plot, based on (48).

\section{Analytical, Simulation and Experimental RESULTS}

In this Section, analytical results based on the continuous and the discrete LTP eigenvalue analysis, as well as timedomain simulations of the switching model and experimental results based on a $10 \mathrm{~kW}$ 2-level IGBT inverter are presented. The validation of the proposed analytical methods will focus on the identification of a stability threshold value, $I_{\text {ref }}^{\text {th }}$, which is the maximum amplitude for the reference inverter current, such that below this value the system in stable and above which the system becomes unstable. The instability threshold has been calculated for different values of the damping resistor $R_{c}$, and as a function of the grid inductor $L_{g}$. The experimental validation has been done in three selected cases as reported in 
Table II, that also anticipates the theoretical stability thresholds $I_{\text {ref }}^{\text {th }}$ that will be derived in the next section for the Continuous and the Discrete LTP model. For all of them, a close match between analytical derivation and experimental evidence has been found.

TABLE II: Experimental test cases

\begin{tabular}{|c|c|c|c|c|}
\hline & $L_{g}$ & $R_{c}$ & $I_{r e f}^{t h}-$ Cont. & $I_{r e f}^{t h}-$ Disc. \\
\hline CASE A & $2.95 m H$ & $1.4 \Omega$ & $9.6 A$ & $9.5 A$ \\
\hline CASE B & $2.2 m H$ & $0.6 \Omega$ & $11.5 A$ & $11.6 A$ \\
\hline CASE C & $2.2 m H$ & $1.2 \Omega$ & $13.1 A$ & $13 A$ \\
\hline
\end{tabular}

\section{A. Continuous LTP Analytical Results}

The eigenvalue loci based on the linearised model (35), with a truncation of the Toeplitz matrix at $N=40$, is evaluated in order to assess the stability of the system. Referring to Table II, the continuous LTP eigenvalue loci plots are reported in Fig.3 for CASE A, where sub-figures $(a)$ and $(b)$ represent $I_{\text {ref }}=$ $9.4 A$, which gives a stable system since all the important eigenvalues lie in the left-hand side of the complex plane, and sub-figures $(c)$ and $(d)$ represent $I_{\text {ref }}=9.8 \mathrm{~A}$, which gives an unstable system, since some of the important eigenvalues lie in the right-hand side of the complex plane. Thus it can be found that $I_{r e f}^{t h}=9.6 A$. Fig. 4 reports the calculated $I_{r e f}^{t h}$ for the set of parameters $R_{c} \in[0.4: 1.6] \Omega$ and $L_{g} \in[2: 3.2] \mathrm{mH}$. The thresholds corresponding to the three cases that will be later validated experimentally have been labelled in figure. The accuracy of the stability analysis depends on the truncation order, which has been chosen based on the results presented in Fig.5, where the percentage error refers to the difference between the stability thresholds calculated using $N \in[2: 30]$ and the one using $N=100$. It can be seen that for $N>20$ the instability threshold $I_{r e f}^{t h}$ is identified with negligible error, hence $N=40$ has been chosen. This is due to the fact that for small $N$ the vertical line of eigenvalues are shifted from their correct location. Some spurious eigenvalues can be seen in Fig.3. They have no physical meaning and they can be easily identified in the eigenvalue plot because they move out of the vertical lines corresponding to the translated copies of the main eigenvalues shown in Fig.2. In fact, the continuous LTP theory is based on Toeplitz forms that involve infinite harmonic terms, but the application of the truncation compromises this assumption and spurious eigenvalues arise.

From Fig.5 it can be clearly observed that the error is still acceptable, i.e. of the order of a few $\%$, also for a truncation order $N=8$, much lower than the one used in the proposed analysis. $N=40$ has been preferred in this paper only to demonstrate the potentially high precision of the method.

\section{B. Discrete LTP Analytical Results}

The eigenvalue loci based on the linearised model (51) is evaluated in order to assess the stability of the system. Referring to Table II, the discrete LTP eigenvalue loci plots are reported in Fig. 6 for CASE A, where the first one is with $I_{r e f}=9.4 \mathrm{~A}$, which gives a stable system since all the eigenvalues lie inside the unit circle, and the second one with $I_{\text {ref }}=9.8 \mathrm{~A}$, which gives an unstable system, since some of

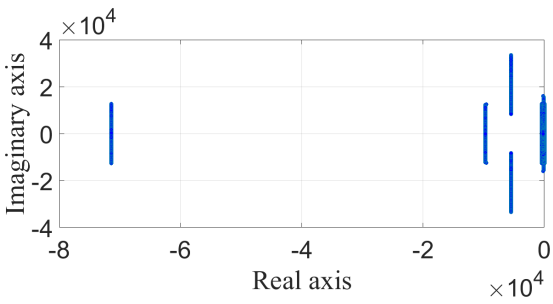

(a)

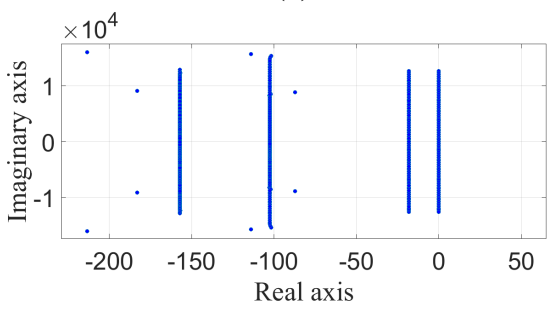

(b)

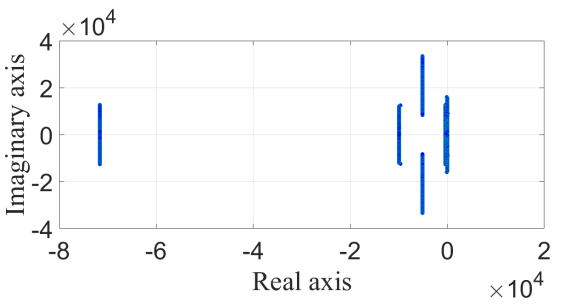

(c)

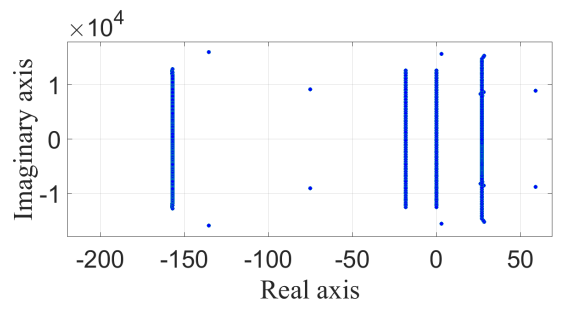

(d)

Fig. 3. CASE A - Continuous LTP Eigenvalues: (a) stable system with $I_{r e f}=9.4 \mathrm{~A}$, (b) zoom around the imaginary axis, (c) unstable system with $I_{r e f}=9.8 \mathrm{~A}$, (d) zoom around the imaginary axis.

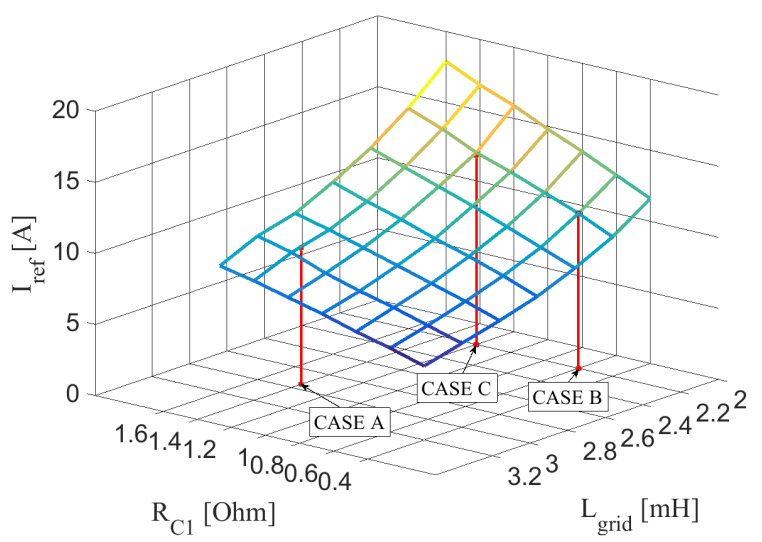

Fig. 4. Continuous LTP: stability threshold $I_{r e f}^{t h}$ as function of $L_{g}$ and $R_{c}$. Red lines indicate stability thresholds that will be validated experimentally, according to Table II.

the eigenvalues lie outside of the unit circle. Thus it can be found that $I_{r e f}^{t h}=9.6 \mathrm{~A}$. Fig.7 reports the calculated $I_{r e f}^{t h}$ for 


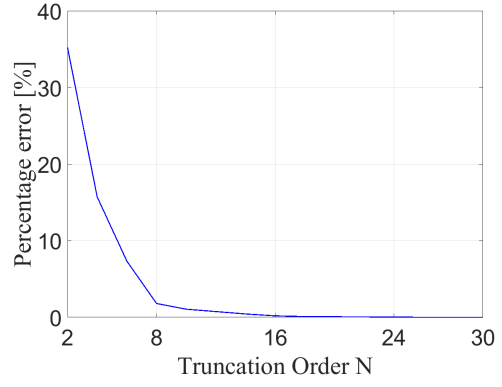

Fig. 5. Stability threshold $I_{r e f}^{t h}$ identification error as a function of the truncation order (Threshold calculated with $N=100$ is considered accurate.)

the set of parameters $R_{c} \in[0.4: 1.6] \Omega$ and $L_{g} \in[2: 3.2] \mathrm{mH}$. The thresholds corresponding to the three cases that will be later validated experimentally have been labeled in the figure.

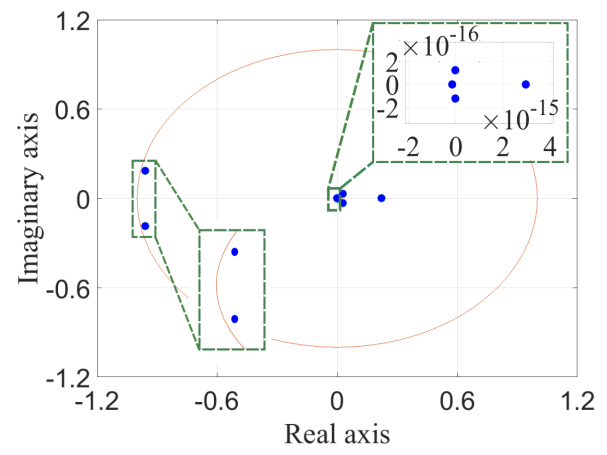

(a)

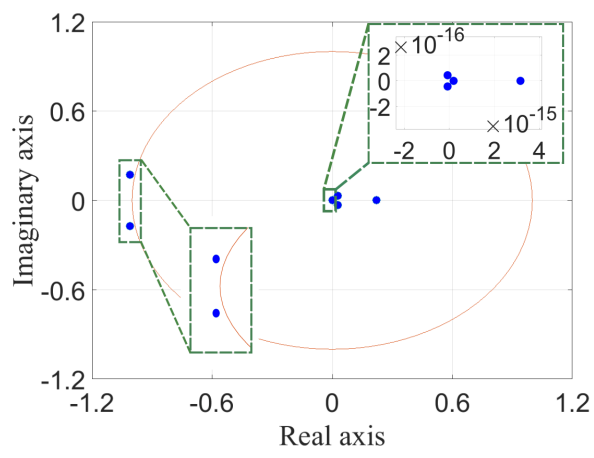

(b)

Fig. 6. CASE A - Discrete LTP Eigenvalues: (a) stable system with $I_{r e f}=9.4 \mathrm{~A}$, (b) unstable system with $I_{r e f}=9.8 \mathrm{~A}$

\section{Time-Domain Simulation Results}

The simulations have been implemented in Matlab Simulink and Plecs toolbox. For brevity, only CASE A from Table II has been considered. The analysis has been performed on the switching model of the system, with control algorithm implemented in C. Moreover, the duty cycle $d(k)$ takes values in the range $[-1,1]$, while $d_{1}(k)$ and $d_{2}(k)$ take values in the range $[0,1]$ (Fig.1), due to the modulation scheme implemented with a single unipolar triangular carrier of frequency $f_{p w m}$ compared compared with the two duty cycles to generate

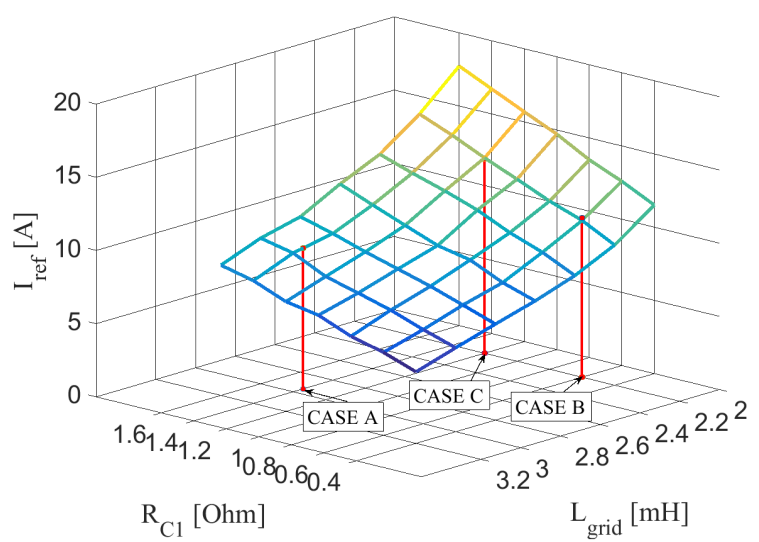

Fig. 7. Discrete LTP: stability threshold $I_{\text {ref }}^{\text {th }}$ as function of $L_{g}$ and $R_{c}$. Red lines indicate stability thresholds that will be validated experimentally, according to Table II.

the gate commands for the two legs. A double update mode is used for the PWM, and therefore the control algorithm is executed at twice the switching frequency, i.e. $f_{x}=2 f_{\text {pwm }}$. Since the IGBTs used in the experimental converter require a non negligible dead time $\left(T_{\text {dead }}=3.2 \mu s\right)$, a standard feedforward compensation term is added to the signal $d(k)$, which provides a compensation to the average value of the output voltage. Such a term, $\operatorname{sign}\left(I_{L 2}(t)\right) 2 T_{\text {dead }} / T_{\text {pwm }}$, depends on the sign of the output current $I_{L 2}(t)$ and a dead band of $\pm 0.5 \mathrm{~A}$ is introduced to deal with zero crossings. $V_{o}(k)$ and $I_{L 2}(k)$ are acquired at discrete times $k T_{x}$, while the output control signals $d_{1}(k)$ and $d_{2}(k)$ are provided by the DSP at discrete times $(k+1) T_{x}$, due to the computation delay time. In Fig. 8 results of the time-domain simulation, showing converter current, capacitor voltage and phase estimated by the PLL are presented, for operation below and above the estimated stability threshold, identified as $I_{\text {ref }}^{\text {th }}=9.6 \mathrm{~A}$ by the theoretical analysis. The small distortion that can be observed in the voltage and current waveforms in the stable case is due to the non-ideal compensation of the dead-times of the IGBTs, by the resonance of the LCL filter and by the fact that the system is operating very close to the instability boundary. It is worth noticing that in Fig. 8(d) the inductor current $I_{L 2}(t)$, within the limits of the control bandwidth, correctly tracks the reference current $I_{r e f}(t)$, confirming that the observed instability is not due to the current control but to the PLL. These simulation results provide a first validation of the accuracy of the stability boundary prediction provided by the LTP eigenvalue analysis.

\section{Experimental Results}

The experimental rig is a $10 k W$ 2-level IGBT inverter switching at $10 \mathrm{kHz}$ and with control algorithms and signal conditioning implemented in a custom DSP/FPGA board. All the relevant parameters of the experimental setup can be found in Table I and Table II. Fig.10 shows a picture of the experimental setup. In Fig.9 converter current, capacitor voltage and phase estimated by the PLL for CASE A are recorded by logging in Matlab the samples measured by the DSP in the experimental setup. A good agreement can be 


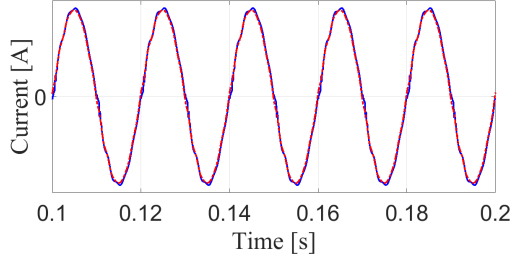

(a)

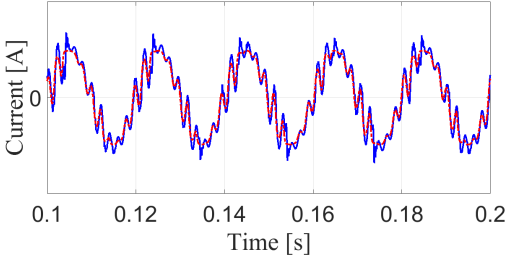

(d)

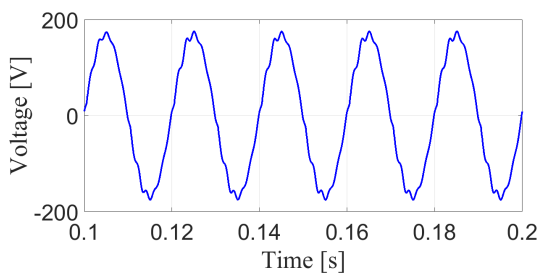

(b)

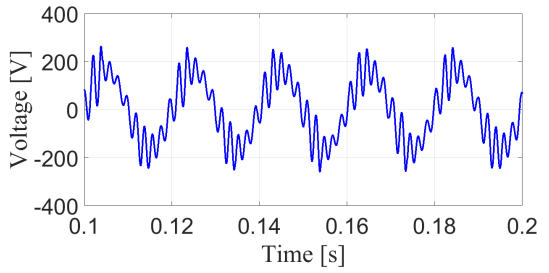

(e)

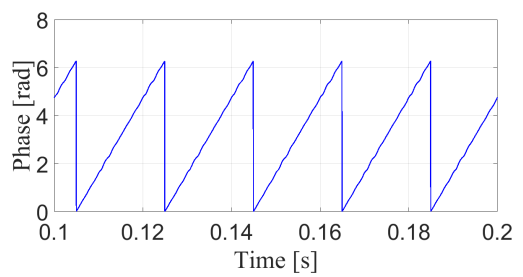

(c)

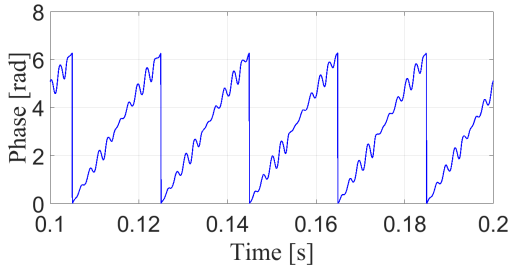

(f)

Fig. 8. Simulation results for CASE A - currents: blue - $I_{L 2}(t)$, red - $I_{r e f}(t)$; voltages: blue - $V_{o}(t)$; phase: blue - $\theta_{P L L}$; (a), (b), (c) stable system with $I_{r e f}=9.4 A$, (d), (e), (f) unstable system with $I_{r e f}=9.8 \mathrm{~A}$.

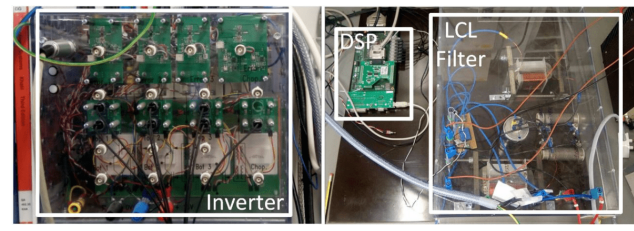

Fig. 10. Single-phase grid-connected inverter with PLL - experimental setup

seen with the time domain simulation reported in Fig.8. In addition, Fig.11, (a) and (d), reports the converter current and capacitor voltage measured with the oscilloscope when the converter is operating below and above the threshold, with $I_{r e f}=9.4 \mathrm{~A}$ and $I_{r e f}=9.8 \mathrm{~A}$ respectively. The same waveforms are measured and reported in Fig.11, (b) and (e), for CASE B, with $I_{r e f}=11.3 \mathrm{~A}$ and $I_{r e f}=11.7 \mathrm{~A}$, and Fig.11, (c) and (f), with $I_{\text {ref }}=12.9 \mathrm{~A}$ and $I_{\text {ref }}=13.3 \mathrm{~A}$. A good agreement with the theoretical results presented in Fig.4 and Fig.7 can be observed.

As a further validation of the threshold for CASE A, Fig.12 shows converter current and capacitor voltage, recorded by logging in Matlab the samples measured by the DSP in the experimental setup, when the amplitude of the reference current $I_{\text {ref }}$ changes with time following a triangular envelope with period $3.5 \mathrm{~s}$ and minimum and maximum values respectively 8.5A and 11A. The converter current and capacitor voltage are stable for $I_{\text {ref }}<9.6 \mathrm{~A}$ and are unstable for $I_{\text {ref }}>9.6 \mathrm{~A}$. The experimental results agree with the theoretical ones, corroborating the validity of the proposed stability analysis method.

\section{COMPARISON AND DISCUSSION}

From the theoretical analysis it can be seen that the continuous and the discrete LTP models both enable an accurate evaluation of the stability boundaries of the system. In fact, the difference between the calculated thresholds with the two approaches is around $3-4 \%$. So the stability assessment can be accurately performed in both continuous and discrete timedomains. However, some features can be discussed as follows:

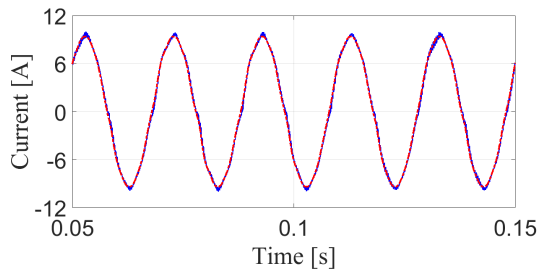

(a)

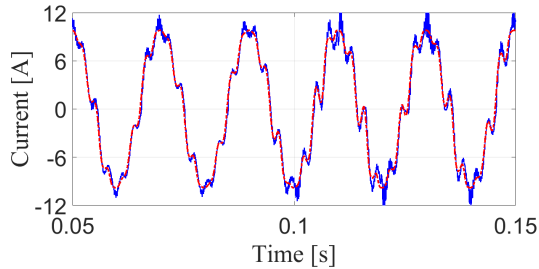

(d)

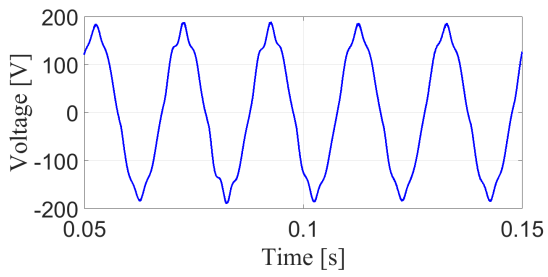

(b)

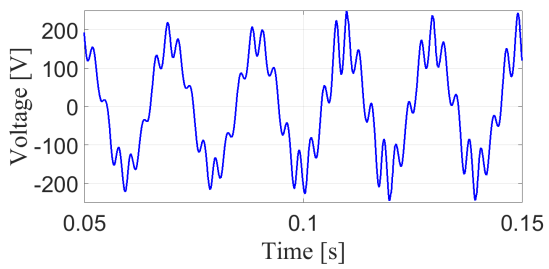

(e)

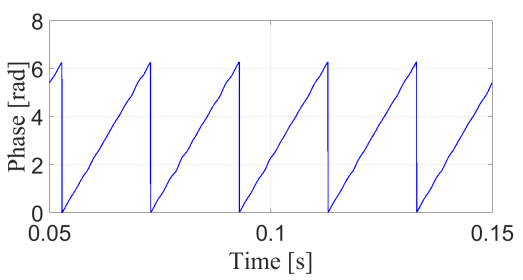

(c)

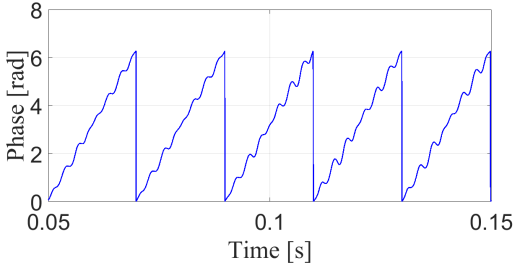

(f)

Fig. 9. Experimental results for CASE A - currents: blue - $I_{L 2}(t)$, red - $I_{r e f}(t)$; voltages: blue - $V_{o}(t)$; phase: blue - $\theta_{P L L}$; (a), (b), (c) stable system with $I_{r e f}=9.4 \mathrm{~A}$, (d), (e), (f) unstable system with $I_{r e f}=9.8 \mathrm{~A}$ - the figure reports data sampled by the controller at $20 \mathrm{kHz}$. 


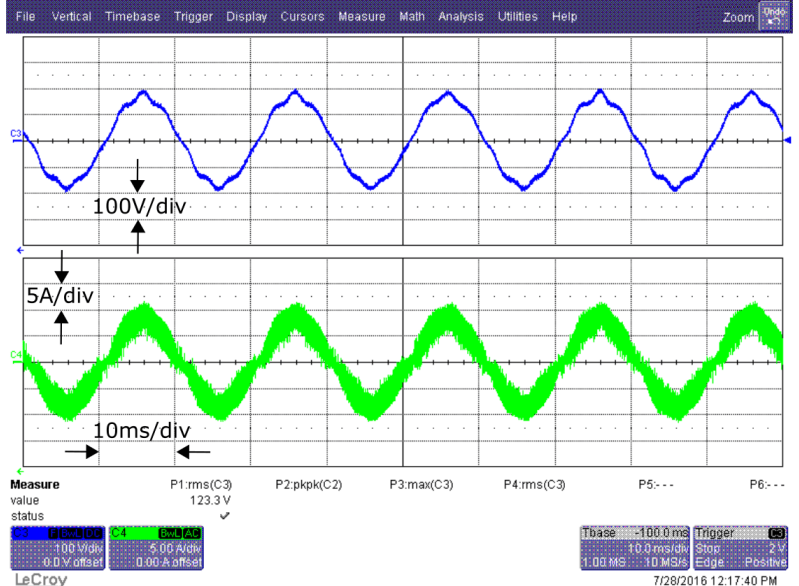

(a)

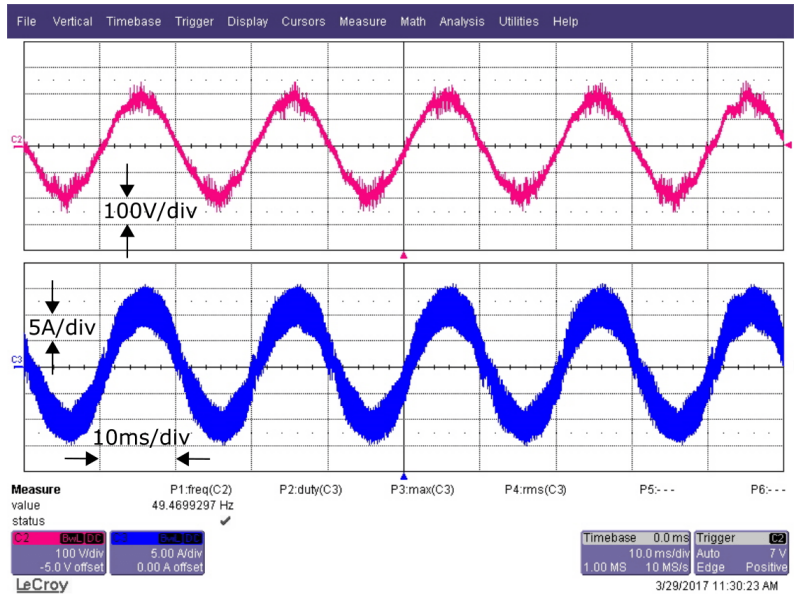

(b)

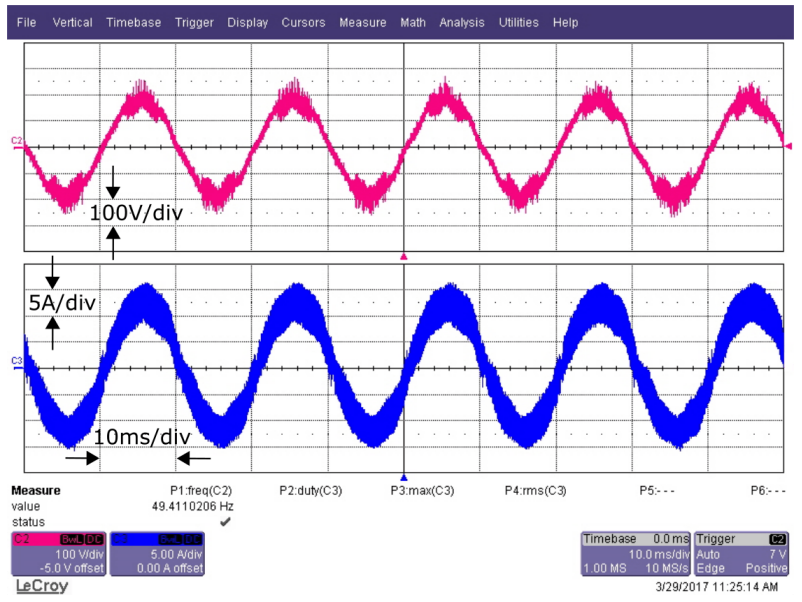

(c)

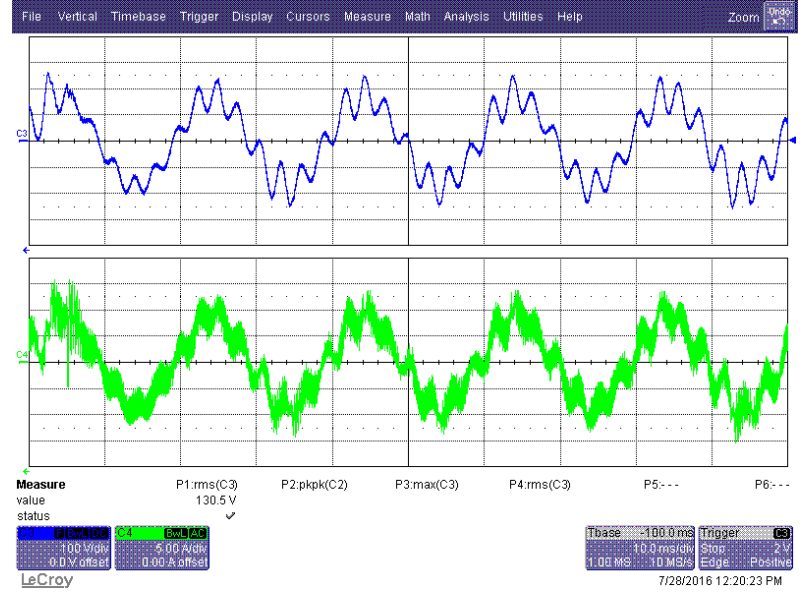

(d)
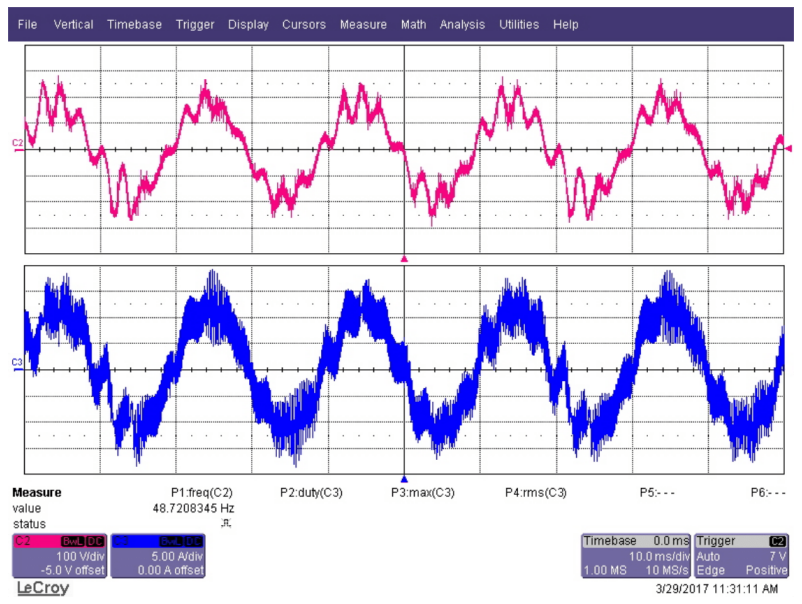

(e)
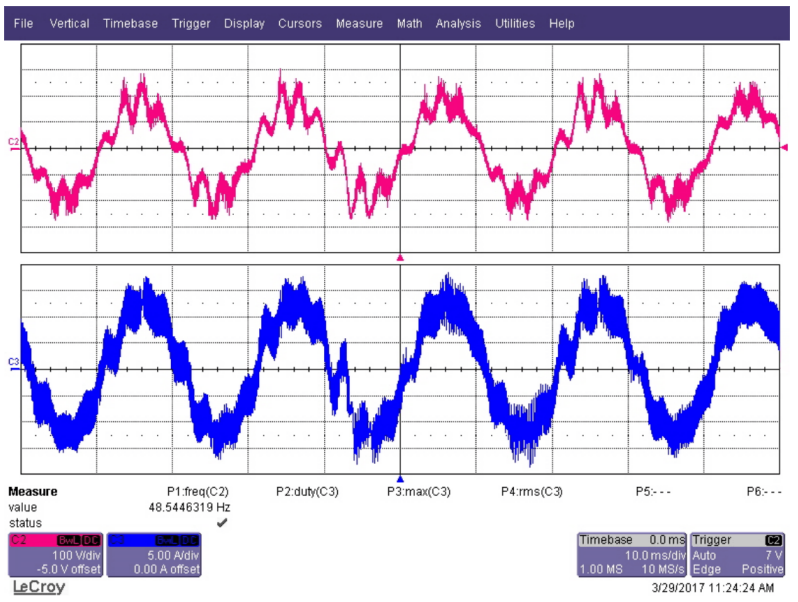

(f)

Fig. 11. CASE A - (a), stable system with $I_{r e f}=9.4 A$, (d), unstable system with $I_{r e f}=9.8 A$; CASE B - (b), stable system with $I_{r e f}=11.3 A$, (e), unstable system with $I_{r e f}=11.7 A$; CASE C - (c), stable system with $I_{r e f}=12.9 A$, (f), unstable system with $I_{r e f}=13.3 A$; top trace: inverter current $I_{L 2}(t)$, bottom trace: voltage $V_{o}(t)$.

- Continuous LTP: the digital computational delay plus $\mathrm{ZOH}$ of the digital PWM can be approximated exploiting the Padé functions. No approximations are made to describe the dynamics of the grid and the filter subsys- tem. Attention must be paid to choose a high enough truncation order, to achieve accurate results.

- Discrete LTP: the control subsystem included in the analysis is the same one implemented on the DSP, and 


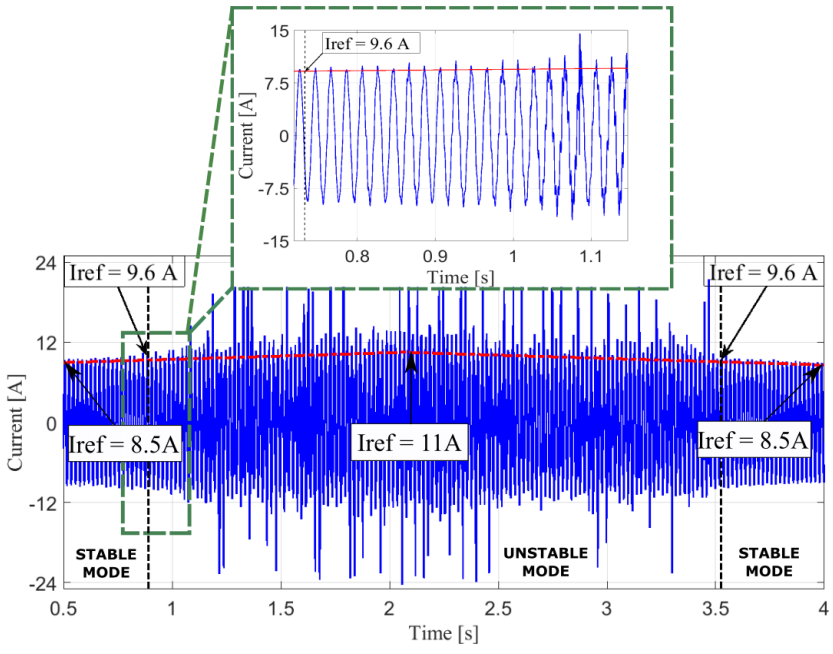

(a)

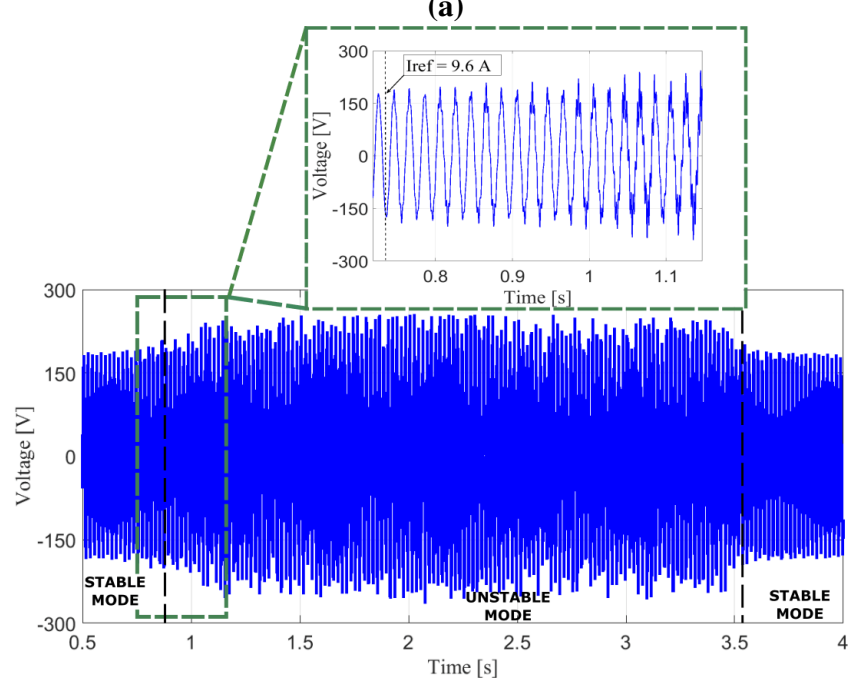

(b)

Fig. 12. CASE A - (a) Currents: blue - $I_{L 2}(t)$, red - $I_{\text {ref }}$ amplitude. (b) Voltages: blue - $V_{o}(t)$.

the digital computational delays are naturally represented in discrete time. The filter-grid continuous part must be approximated by a discrete model. No considerations related to the order of the system and truncations are required, since in this case the stability analysis relies on a different theoretical approach.

\section{CONCLUSION}

In this paper a general method is presented, based on LTP theory, to perform stability analysis of power converters with non-linear control and time-periodic operating trajectories, thus providing a technique to precisely analyse single phase or heavily unbalanced three phase systems. The proposed analysis can be performed both in the continuous and in the discrete time-domain. The paper shows how the two methods, despite a different theoretical background, can identify the stability threshold with comparable precision. The objective of the analysis is to capture effects of the non-linear dynamics that would not be detectable with simplified low frequency averaging and LTI analysis. Validity of the model for high frequencies is limited by the averaging approach employed to represent the inverter. The method has been applied to a singlephase grid-connected inverter with PLL, showing how stability depends on the amplitude of the grid current reference. For a given set of converter and grid parameters, current controller design and PLL design, with the proposed approach it is possible to derive analytically the maximum current reference that guarantees stable operation. Numerical simulations and experiments on a $10 \mathrm{~kW}$ prototype have been performed to validate the proposed stability analysis technique, including different values of grid inductor and damping resistor, always showing a good match between the predicted stability boundaries and those measured in the experimental setup.

\section{REFERENCES}

[1] E. Mollerstedt and B. Bernhardsson, "Out of control because of harmonics-an analysis of the harmonic response of an inverter locomotive," IEEE Control Systems, vol. 20, pp. 70-81, Aug 2000.

[2] E. Mollerstedt and B. Bernhardsson, "A harmonic transfer function model for a diode converter train," in Power Engineering Society Winter Meeting, 2000. IEEE, vol. 2, pp. 957-962 vol.2, 2000.

[3] H. Liu and J. Sun, "Modeling and analysis of dc-link harmonic instability in LCC HVDC systems," in Control and Modeling for Power Electronics (COMPEL), 2013 IEEE 14th Workshop on, pp. 1-9, June 2013.

[4] M. Bollen, J. Meyer, H. Amaris, A. M. Blanco, A. G. de Castro, J. Desmet, M. Klatt, L. Kocewiak, S. Rönnberg, and K. Yang, "Future work on harmonics - some expert opinions part I - wind and solar power," in 2014 16th International Conference on Harmonics and Quality of Power (ICHQP), pp. 904-908, May 2014.

[5] J. Meyer, M. Bollen, H. Amaris, A. M. Blanco, A. G. de Castro, J. Desmet, M. Klatt, L. Kocewiak, S. Rönnberg, and K. Yang, "Future work on harmonics - some expert opinions part II - supraharmonics, standards and measurements," in 2014 16th International Conference on Harmonics and Quality of Power (ICHQP), pp. 909-913, May 2014.

[6] B. Wen, D. Boroyevich, R. Burgos, P. Mattavelli, and Z. Shen, "Analysis of d-q small-signal impedance of grid-tied inverters," IEEE Transactions on Power Electronics, vol. 31, pp. 675-687, Jan 2016.

[7] J. Sun, "Impedance-based stability criterion for grid-connected inverters," IEEE Transactions on Power Electronics, vol. 26, pp. 3075-3078, Nov 2011.

[8] J. Sun and K. Karimi, "Small-signal input impedance modeling of linefrequency rectifiers," Aerospace and Electronic Systems, IEEE Transactions on, vol. 44, pp. 1489-1497, Oct 2008.

[9] J. Sun, "Input impedance analysis of single-phase pfc converters," Power Electronics, IEEE Transactions on, vol. 20, pp. 308-314, March 2005.

[10] V. Salis, A. Costabeber, P. Zanchetta, and S. Cox, "A generalised harmonic linearisation method for power converters input/output impedance calculation," in 2016 18th European Conference on Power Electronics and Applications (EPE'16 ECCE Europe), pp. 1-7, Sept 2016.

[11] J. Sun, "Small-signal methods for ac distributed power systems 2013; a review," Power Electronics, IEEE Transactions on, vol. 24, pp. 25452554, Nov 2009.

[12] S. Shah and L. Parsa, "On impedance modeling of single-phase voltage source converters," in 2016 IEEE Energy Conversion Congress and Exposition (ECCE), pp. 1-8, Sept 2016.

[13] S. Lissandron, L. D. Santa, P. Mattavelli, and B. Wen, "Experimental validation for impedance-based small-signal stability analysis of singlephase interconnected power systems with grid-feeding inverters," IEEE Journal of Emerging and Selected Topics in Power Electronics, vol. 4, pp. 103-115, March 2016.

[14] A. G. J. MacFarlane and I. Postlethwaite, "The generalized Nyquist stability criterion and multivariable root loci," International Journal of Control, vol. 25, no. 1, pp. 81-127, 1977.

[15] N. M. Wereley and S. R. Hall, "Frequency response of linear time periodic systems," in Decision and Control, 1990., Proceedings of the 29th IEEE Conference on, pp. 3650-3655 vol.6, Dec 1990.

[16] S. R. Hall and N. M. Wereley, "Generalized Nyquist stability criterion for linear time periodic systems," in American Control Conference, 1990, pp. 1518-1525, May 1990. 
[17] J. R. C. Orillaza and A. R. Wood, "Harmonic state-space model of a controlled TCR," IEEE Transactions on Power Delivery, vol. 28, pp. 197-205, Jan 2013.

[18] M. S. P. Hwang and A. R. Wood, "A new modelling framework for power supply networks with converter based loads and generators the harmonic state-space," in Power System Technology (POWERCON), 2012 IEEE International Conference on, pp. 1-6, Oct 2012.

[19] J. Kwon, X. Wang, C. L. Bak, and F. Blaabjerg, "Harmonic instability analysis of single-phase grid connected converter using harmonic state space (HSS) modeling method," in 2015 IEEE Energy Conversion Congress and Exposition (ECCE), pp. 2421-2428, Sept 2015.

[20] J. Kwon, X. Wang, C. L. Bak, and F. Blaabjerg, "Harmonic interaction analysis in grid connected converter using harmonic state space (HSS) modeling," in Applied Power Electronics Conference and Exposition (APEC), 2015 IEEE, pp. 1779-1786, March 2015.

[21] R. Z. Scapini, L. V. Bellinaso, and L. Michels, "Stability analysis of half-bridge rectifier employing LTP approach," in IECON 2012 - 38th Annual Conference on IEEE Industrial Electronics Society, pp. 780-785, Oct 2012.

[22] L. V. Bellinaso, R. Z. Scapini, and L. Michels, "Modeling and analysis of single phase full-bridge PFC boost rectifier using the LTP approach," in XI Brazilian Power Electronics Conference, pp. 93-100, Sept 2011.

[23] C. Zou, B. Liu, S. Duan, and R. Li, "Influence of delay on system stability and delay optimization of grid-connected inverters with lcl filter," IEEE Transactions on Industrial Informatics, vol. 10, pp. 17751784, Aug 2014.

[24] C. Zhang, X. Wang, and F. Blaabjerg, "Analysis of phase-locked loop influence on the stability of single-phase grid-connected inverter," in 2015 IEEE 6th International Symposium on Power Electronics for Distributed Generation Systems (PEDG), pp. 1-8, June 2015.

[25] V. Salis, A. Costabeber, P. Zanchetta, and S. Cox, "Stability analysis of single-phase grid-feeding inverters with pll using harmonic linearisation and linear time periodic (ltp) theory," in 2016 IEEE 17th Workshop on Control and Modeling for Power Electronics (COMPEL), pp. 1-7, June 2016.

[26] D. M. Van de Sype, K. De Gusseme, A. P. Van den Bossche, and J. A. Melkebeek, "Small-signal Laplace-domain analysis of uniformlysampled pulse-width modulators," in Power Electronics Specialists Conference, 2004. PESC 04. 2004 IEEE 35th Annual, vol. 6, pp. 4292-4298 Vol.6, June 2004.

[27] S. Buso and P. Mattavelli, Digital Control in Power Electronics, 2nd Edition. Morgan \& Claypool, 2015.

[28] R. Meyer and C. Burrus, "A unified analysis of multirate and periodically time-varying digital filters," IEEE Transactions on Circuits and Systems, vol. 22, pp. 162-168, Mar 1975.

[29] S. Bittanti and P. Colaneri, "Invariant representations of discrete-time periodic systems," Automatica, vol. 36, no. 12, pp. 1777 - 1793, 2000.

[30] Z. Bing, K. Karimi, and J. Sun, "Input impedance modeling and analysis of line-commutated rectifiers," Power Electronics, IEEE Transactions on, vol. 24, pp. 2338-2346, Oct 2009.

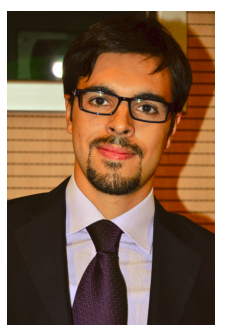

Valerio Salis received the Master's degree with honours in Electronic Engineering from the University of Rome Tor Vergata, Rome, Italy, in 2014. He is currently working towards the Ph.D. degree in Electrical and Electronic Engineering in the Power Electronics, Machines and Control Group, University of Nottingham, Nottingham, UK. His research interests include study of instability issues in microgrids, linear time periodic system analysis and control design.

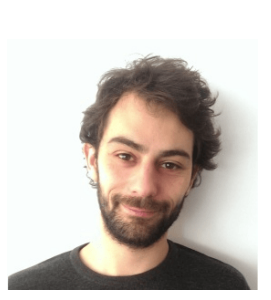

Alessandro Costabeber (S'09 - M'13) received the Degree with honours in Electronic Engineering from the University of Padova, Padova, Italy, in 2008 and the Ph.D. in Information Engineering from the same university in 2012, on energy efficient architectures and control techniques for the development of future residential microgrids. In the same year he started a two-year research fellowship with the same university. In 2014 he joined the PEMC group, Department of Electrical and Electronic Engineering, University of Nottingham, Nottingham, UK as Lecturer in Power Electronics. His current research interests include HVDC converters topologies, high power density converters for aerospace applications, control solutions and stability analysis of AC and DC microgrids, control and modelling of power converters, power electronics and control for distributed and renewable energy sources. Dr. Costabeber received the IEEE Joseph John Suozzi INTELEC Fellowship Award in Power Electronics in 2011.

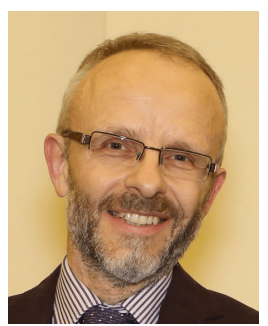

Stephen M. Cox received the B.A. degree in mathematics from the University of Oxford, Oxford, U.K., in 1986, and the Ph.D. degree in applied mathematics from the University of Bristol, Bristol, U.K., in 1989. He is currently a Reader in the School of Mathematical Sciences, University of Nottingham, Nottingham, U.K., where he was also a Lecturer and then a Senior Lecturer. From 2004 to 2006, he was a Senior Lecturer in the School of Mathematical Sciences, University of Adelaide, Australia. His research interests include the development of applied mathematical techniques for the modeling of class-D amplifiers and other power-electronic switching devices.

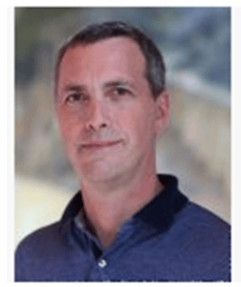

Pericle Zanchetta (M'00 - SM'15) received his MEng degree in Electronic Engineering and his $\mathrm{Ph} . \mathrm{D}$. in Electrical Engineering from the Technical University of Bari (Italy) in 1993 and 1997 respectively. In 1998 he became Assistant Professor of Power Electronics at the same University. In 2001 he became lecturer in control of power electronics systems in the PEMC research group at the University of Nottingham UK, where he is now Professor in Control of Power Electronics systems. He has published over 260 peer reviewed papers and he is Chair of the IAS Industrial Power Converter Committee IPCC. His research interests include control of power converters and drives, Matrix and multilevel converters.

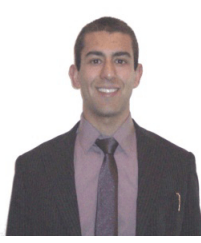

Andrea Formentini was born in Genova, Italy, in 1985. He received the M.S. degree in computer engineering and the Ph.D. degree in electrical engineering from the University of Genova, Genova, in 2010 and 2014 respectively. He is currently working as research fellow in the Power Electronics, Machines and Control Group, University of Nottingham. His research interests include control systems applied to electrical machine drives and power converters. 